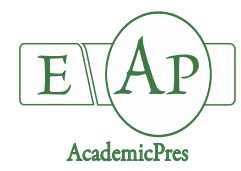

Al-Elwany OAAI et al. (2020)
Notulae Botanicae Horti Agrobotanici Cluj-Napoca 48(4):1957-1979
DOI:10.15835/48412126

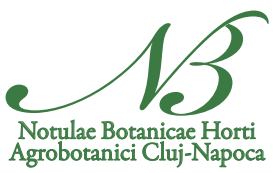

\title{
Exogenous glutathione-mediated tolerance to deficit irrigation in salt- affected Capsicum frutescence (L.) plants is connected with higher antioxidant content and ionic homeostasis
}

\author{
Omar A.A.I. AL-ELWANY ${ }^{1}$, Gamal F. MOHAMED², Hamdi A. \\ ABDURRAHMAN ${ }^{3}$, Mostafa M. RADY ${ }^{2}$, Arafat A. ABDEL LATEF ${ }^{4,5 *}$ \\ ${ }^{1}$ Fayoum University, Faculty of Agriculture, Horticulture Department, Fayoum 63514,Egypt; oaa00@fayoum.edu.eg \\ ${ }^{2}$ Fayoum University, Faculty of Agriculture, Botany Department, Fayoum 63514, \\ Egypt;gfm00@fayoum.edu.eg:mmr02@fayoum.edu.eg \\ ${ }^{3}$ Fayoum University, Faculty of Agriculture, Soil and Water Department, Fayoum 63514,Egypt; haaO1@fayoum.edu.eg \\ ${ }^{4}$ Turabah University College, Turabah Branch, Department of Biology, Taif University, P.O. Box 11099, Taif 21944, \\ Saudi Arabia; a.moawd@tu.edu.sa \\ ${ }^{5}$ South Valley University, Faculty of Science, Botany and Microbiology Department, Qena 83523, \\ Egypt;moawad76@gmail.com (*correspondingauthor); a.moawd@tu.edu.sa
}

\begin{abstract}
As an important medicinal plant used in traditional and modern medicine, chili peppers are sensitive or moderately sensitive to drought or salt stress, respectively. Therefore, potential changes due to foliar-applied glutathione (GSH; 0, 0.4 and $0.8 \mathrm{mM}$ ) response on growth, yield, and physio-biochemical attributes, as well as water use efficiency (WUE) and fruit alkaloid capsaicin of chili pepper plants were investigated when grown under deficit irrigation in salt-affected soil $\left(\mathrm{EC}=6.74 \mathrm{dS} \mathrm{m} \mathrm{m}^{-1}\right)$. Two deficit irrigation water $(\mathrm{DiW})$ regimes ( $80 \%$ and $60 \%$ of soil field capacity; FC) were used versus $100 \%$ of FC as a control. Both DiW treatments negatively affected growth and yield parameters, SPAD chlorophyll index, nutrient status, $\mathrm{K}^{+} / \mathrm{Na}^{+}$ratio, and plant anatomical features. In contrast, osmoprotectants, ascorbate, glutathione, capsaicin, and phenolic contents, as well as WUE were increased in association with higher $\mathrm{Na}^{+}$and $\mathrm{Cl}^{-}$contents. However, exogenously-applied GSH caused significant increases in the above-mentioned parameters along with an additional increase in osmoprotectants, antioxidants, and capsaicin contents, and a decrease in $\mathrm{Na}^{+}$and $\mathrm{Cl}^{-}$ levels compared to corresponding controls. The highest WUE, growth, and fruit yield responses were recorded at $0.8 \mathrm{mM}$ GSH applied to plants under DiW at $80 \% \mathrm{FC}+$ salinity $\left(6.74 \mathrm{dS} \mathrm{m} \mathrm{m}^{-1}\right)$. Therefore, this study suggested the use of leafy-applied GSH at $0.8 \mathrm{mM}$ for satisfactory growth and yield with the highest WUE of chili pepper plants grown under salt-affected conditions with deficit irrigation.
\end{abstract}

Keywords: anatomy; capsaicin; chili pepper; environmental stresses; exogenous antioxidant applications; nutrient 


\section{Introduction}

During their life cycles, higher plants always face environmental stress factors, the most dangerous of which are the shortage of fresh water for irrigation and soil salinity. Deficiency of irrigation water (DiW) and soil salinity stress (SsS) solely or in combination severely affect the growth and productivity of most plant species (Abd El-Mageed et al., 2018; Yang et al., 2019; Abid et al., 2020). As freshwater is the most important resource and a limiting factor for agricultural development, its diminishing availability has become a global problem that requires the development of alternative resources for agricultural use. Therefore, practices that increase water use efficiency (WUE) and reduce the amount of added water are important for water conservation. Besides, the expected soil salinization due to $\mathrm{DiW}$ must also be addressed by applying useful techniques in this regard.

Through some efforts, plants are participating in a series of changes in morphology, physiology, biochemistry, and molecular biology to overcome such adversities from these stressors. Understanding the response of plants to combined stressors is unavoidable to improve plant adaptations under open field conditions (Abd El-Mageed et al., 2018; Desoky et al., 2019; Rady et al., 2019; Alharby et al., 2020). In particular, SsS and DiW are often combined either due to the presence of salt naturally in the soil or to an increase in its accumulation due to a lack of salt washing as a result of a DiW. Thus, the low washing of salts from agricultural land due to the $\mathrm{DiW}$ is one of the main reasons for increasing soil salinization. Besides the DiW, poor management of available water in a hot climate of low-rainfall agricultural regions also contributes to soil salinization (Tester and Bacic, 2005; Yang et al., 2019; Alharby et al., 2020; Osman et al., 2020). Therefore, to prevent the loss of agricultural products, more research should be developed to adopt uncomplicated techniques for agricultural producers to use for stressful crop plants to cope with stresses that worsen day by day.

In a similar fashion to DiW, plants respond to SsS. Like drought, osmotic mechanisms related to SsS and restricted the availability of water and thus decreased plant growth along with cellular metabolic alterations (Munns, 2002; Abid et al., 2020). The SsS and/or DiW strictly reduces the plant's ability to use water, which disrupts cell water content and turgor. Cell enlargement, nutrient homeostasis, photosynthetic machinery, and other metabolic processes are also disrupted due to these two stresses. Also, enzyme catalysts including Rubisco enzymes are inhibited, and specific toxic (e.g., $\mathrm{Na}^{+}$and $\mathrm{Cl}^{-}$) ions are raised, and plant death is finally unavoidable (Munns, 2002; Farooq et al., 2017; Hussain et al., 2018; Alharby et al., 2020). SsS threatens the existence of plants, especially those sensitive to high salt levels (Horie et al., 2011). Regardless of whether DiW is present, SsS causes "physiological drought" and ion toxicity, affecting plant growth, yield, photosynthesis, osmoprotectants, antioxidants, nutrient status, anatomy, and WUE (Abd El-Mageed et al., 2018; Shahid et al., 2018; Alharby et al., 2020). Additionally, SsS-DiW-plant relations often restrict cellular normal physiological activities and the productive capacity of crop plants (Alharby et al., 2020).

To cope with stress, plants develop and/or adopt the preservative antioxidant system, including soluble sugars, free proline, glutathione, ascorbate, phenolic compounds, capsaicin, etc. (Abdel Latef et al., 2017a, b, c, 2018, 2019a, b, 2020; Caliskan et al., 2017; Rady et al., 2019; Alharby et al., 2020; Taha et al., 2020). In most cases, the plant antioxidant system is not sufficient to enable it to withstand high stress (e.g., SsS + DiW). Therefore, exogenous supplementation of antioxidants such as glutathione (GSH) should be used to raise plant tolerance to the combined stress under study (Rady and Hemida, 2016; Hasanuzzaman et al., 2017; El-Beltagi et al., 2020).

Antioxidants protect plants from abiotic stress-induced oxidative damage (Rady and Hemida, 2016; ElBeltagi et al., 2020). Among them, GSH (c-glutamyl-cysteinyl-glycine) is a low molecular weight and watersoluble thiol compound, which is widely distributed in most plant tissues. Regardless of its role in storing and transporting reduced sulfur, GSH is involved in the detoxification of reactive oxygen species - ROS (Foyer and Noctor, 2005a, b). It acts as a co-factor in different biochemical reactions. It interacts with hormones, signaling molecules, and its redox state triggers signal transduction (Foyer and Noctor, 2005a, b). It also plays a vital role 
in detoxifying toxic metals/metalloids and xenobiotics (Srivalli and Khanna-Chopra, 2008). Thus, GSH can participate in plant growth and development under conditions of both normal growth and stress. Exogenouslyapplied GSH can enhance plant tolerance to different abiotic stresses, including salinity and drought (Hasanuzzaman et al., 2017).

Pepper (Capsicum spp.) is one of the most important, most popular, and most favourite vegetable crops cultivated in both greenhouses and open fields worldwide, especially in the warm-climate, including Egypt. Medicinally, fruits of chili pepper (Capsicum frutescence L.) are an excellent source of bioactive products, natural colors, and antioxidant compounds, especially the alkaloid 'capsaicin', which is linked to the plant's response to stressful conditions (Phimchan and Techawongstien, 2012; Kpinkoun et al., 2019). Chili peppers are sensitive or moderately sensitive to drought or salt stress, respectively (Lee, 2006; Ben-Gal et al., 2008; Abdel Latef and Chaoxing, 2014). The growth and productivity of peppers are negatively affected under SsS and DiW (Mardani et al., 2017; Taha et al., 2018; Abd El-Mageed et al., 2020). The fruit alkaloid 'capsaicin' content as an important bioactive compound in peppers has been reported to increase significantly under DiW (Kopta et al., 2020) and SsS (Kpinkoun et al., 2019).

As we know, there are no studies available on the effect of combined stress ( $\mathrm{DiW}+\mathrm{SsS}$ ) on growth, fruit yield, and fruit alkaloid capsaicin content in chili pepper and mitigating the combined stress effects by leafyapplied GSH. Therefore, the main objective of the present study was to evaluate the potential positive impacts of GSH applied as a foliar spray to chili pepper plants stressed with combined SsS and DiW on growth, yield, and fruit capsaicin content. The possibility of reaching the ion homeostasis due to the modification of osmoprotectants and antioxidants, including phenols and capsaicin by the application of GSH was also evaluated under the tested combined stress.

\section{Materials and Methods}

\section{Experimental location, climatic conditions, and soil analysis}

A pot experiment was conducted three times simultaneously in the 2018/2019 summer season using an open greenhouse in the experimental farm (Southeast of Fayoum; $29^{\circ} 17^{\prime} \mathrm{N} ; 30^{\circ} 53^{\prime} \mathrm{E}$ ) at the Faculty of Agriculture, Fayoum University, Egypt. Average climatic conditions throughout the experimental period (March 28-June 28) were $32 \pm 3 / 18 \pm 2{ }^{\circ} \mathrm{C}$ for average day/night temperatures, $65 \pm 4 \%$ for average relative humidity, and $13 \mathrm{~h}$ for average daylight length. For light intensity, natural sunlight was suitable for all chili pepper growth stages. Soil physical and chemical properties (Wilde et al., 1985) indicated that the soil electrical conductivity (EC) was $6.74 \mathrm{dS} \mathrm{m}^{-1}$, indicating that it was saline soil (Dahnke and Whitney, 1988). It is characterized as a loamy clay with $22.4 \%$ for field capacity, 7.57 for $\mathrm{pH}, 1.02 \%$ as organic matter content, 22.4, 25.8 , and $21.1 \mathrm{meq} \mathrm{L}^{-1}$ for $\mathrm{Ca}^{2+}, \mathrm{Na}^{+}$and $\mathrm{Cl}^{-}$concentrations, and $15.9,124.0$, and $372.0 \mathrm{mg} \mathrm{kg}^{-1} \mathrm{dry}$ soil for $\mathrm{N}, \mathrm{P}$, and $\mathrm{K}^{+}$, respectively.

\section{Plant material, experimental layout, and cultural practices}

Chili pepper seeds were obtained from the Agricultural Research Centre (ARC), Giza, Egypt. They were incubated for germination on January 25 in a private nursery, then the standardized transplants (with 2-3 pairs of true expanded leaves) were transplanted into pots ( $40 \mathrm{~cm}$ in diameter, each $12 \mathrm{~kg}$ of air-dried soil) on March 28 at a rate of two transplants per pot. Before transplantation, treatments were arranged in a completely randomized layout in a factorial design. Regardless of the irrigation system as an experimental factor, standard agronomic practices were applied including pest and disease control, and fertilization program as recommended for commercial peppers production. Before transplanting, $2.4 \mathrm{~g}$ of NPK fertilizer $\left(20,20\right.$, and $20 \%$ of $\mathrm{N}, \mathrm{P}_{2} \mathrm{O}_{5}$, and $\mathrm{K}_{2} \mathrm{O}$, respectively) was allocated for each pot (12 kg soil). After transplanting, foliar fertilization was applied once a week, regularly for all plants with $1 \mathrm{~g}$ Kristalon L ${ }^{-1}$ (YARA Agri, Staré Me`sto, Czech Republic), 
which contains $20,5,10$, and $2 \%$ of $\mathrm{N}, \mathrm{P}_{2} \mathrm{O}_{5}, \mathrm{~K}_{2} \mathrm{O}$, and $\mathrm{Mg}$, respectively starting from 20 days after transplanting (DAT) until the end of the flowering stage.

\section{Treatments and other practices}

With saline soil $\left(\mathrm{ECe}=6.74 \mathrm{dS} \mathrm{m}^{-1}\right)$, two factors were applied. Irrigation water was applied until the end of the experiment (28 June) in 3 regimes $(60 \%, 80 \%$, and 100\%) based on soil field capacity (FC) each of 60 pots. The pots for each water regime were divided into three groups, each of 20 pots, intended for one of three levels of GSH; 0 (distilled water as a control), 0.4 , and $0.8 \mathrm{mM}$. Therefore, a total of 9 treatments ( 3 water regimes $\times 3$ GSH levels), all of them applied under combined stress (DiW + SsS; $\left.6.74 \mathrm{dS} \mathrm{m}^{-1}\right)$. The GSH concentrations used in this study were selected based on a preliminary study in which the concentrations of 0 , $0.2,0.4,0.6,0.8,1.0$, and $1.2 \mathrm{mM}$ GSH were applied, and the concentration of $0.8 \mathrm{mM}$ produced the best response (data not shown), therefore, it was chosen along with $0.4 \mathrm{mM}$ for this study.

Once the transplanting was completed, the GSH solutions were sprayed using Dorsal Spray Machine $(20 \mathrm{~L})$. The seedlings were allowed to establish for 14 days after transplantation (DAT), then irrigation water treatments were started. At the same time (14 DAT), the plants were sprayed again with GSH solutions. The spraying was then repeated with GSH solutions at 28 and 42 DAT. To avoid runoff and to ensure penetration of spray solutions into leaf tissues, Tween-20 (1\%) was added to the spraying solutions as a wetting agent. Using time-domain reflectometry (TDR200, Soil Moisture Equipment, Spectrum, USA), monitoring and measuring water deficits were implemented for different water treatments by inserting a waveguide probe with a length of $20 \mathrm{~cm}$ in the upper $20-\mathrm{cm}$ soil. After the daily weight, the pots were supplied with the targeted amount of irrigation water based on the measured DiW and the tested treatments. Depending on the location in the greenhouse, the pots were rotated every $48 \mathrm{~h}$ to ensure fair distribution of daylight and sunlight intensity for all experimental plants.

\section{Growth and yield characteristics}

Ninety DAT, five plants with root systems were randomly selected from each treatment to assess growth characteristics. Plant height was measured, main branches and leaves were counted, and fresh weight of shoot and root systems were recorded. The shoot and root systems were then dried at $70{ }^{\circ} \mathrm{C}$ until a constant dry weight was obtained. Twenty-five plants were dedicated to the chili pepper fruit yield. The fruits were harvested for the average number, fresh and dry weights for each plant, and capsaicin content.

\section{Physio-biochemical analyses}

Ninety DAT, five plants were randomly selected from each treatment for physio-biochemical assessments. Using the fully expanded third upper leaf, the concentration of total chlorophylls was measured as SPAD units using the Minolta chlorophyll meter (SPAD 502 model). Using dry matter of the fully expanded third leaf, the contents of free proline and total soluble sugars were assessed colorimetrically according to the procedures detailed in Bates et al. (1973) and Irigoyen et al. (1992), respectively. Using the methanolic extract of the same leaf material, total phenolic content was determined using the Folin-Ciocalteu colorimetric method described by Singleton and Rossi (1965). Total capsaicin content $\left(\mathrm{mg} \mathrm{g}^{-1} \mathrm{DW}\right)$ in dry fruits was extracted and estimated according to the method described in Augusto and Carloswild (1973). Using the same leaf material, $\mathrm{N}$ content was determined colorimetrically by using the Orange $\mathrm{G}$ dye (Rady, 2012). The content of $\mathrm{P}$ was quantitatively measured using the molybdenum-reduced molybdophosphoric blue color method (Jackson, 1967). The contents of $\mathrm{K}^{+}$and $\mathrm{Na}^{+}$were measured using a Perkin-Elmer Model 52-A Flame Photometer (Wilde et al., 1985). The contents of $\mathrm{Ca}^{2+}, \mathrm{Mg}^{2+}, \mathrm{Fe}^{2+}, \mathrm{Zn}^{2+}, \mathrm{Mn}^{2+}$, and $\mathrm{Cl}^{-}$were measured using a Perkin-Elmer Atomic Absorption Spectrophotometer (Higinbotham et al., 1967). 


\section{Water use efficiency (WUE, \%)}

Irrigation WUE was calculated according to Guang-Cheng et al. (2008). Water-saving (\%) was calculated from the following equation: water-saving $(\%)=[($ water consumption of control - water consumption of treatment)/water consumption of control] $\times 100$.

\section{Anatomical study}

Forty-five DAT, five plants were randomly selected from each treatment for anatomical studies. Stem and leaf samples were taken from the middle of the fourth leaf from the apex. They were killed and fixed in FAA solution $(50 \mathrm{ml}$, $95 \%$ ethyl alcohol $+10 \mathrm{ml}$ formalin $+5 \mathrm{ml}$ glacial acetic acid $+35 \mathrm{ml}$ distilled water $)$ for $48 \mathrm{~h}$. They were then washed in $50 \%$ ethyl alcohol, dehydrated, and cleared in tertiary butyl alcohol series, embedded in paraffin wax of $54-56^{\circ} \mathrm{C}$ m.p. Cross-sections, $20 \mu$ thick, were cut by a rotary microtome, adhered by Haupt's adhesive, stained with the crystal violet-erythrosin combination (Nassar and El-Sahhar, 1998), cleared in carbol-xylene and mounted in Canada balsam. The sections were observed and documented using an upright light microscope (AxioPlan, Zeiss, Jena, Germany). A micrometer eyepiece was used for measurements.

\section{Statistical analysis}

The data are presented as means \pm standard errors (SE). They were subjected to a two-way analysis of variance (ANOVA), and Duncan's Multiple Range Test was used to identify the significant variations among means, comparing at $P \leq 0.05$. The COSTAT computer software (CoHort Software version 6.303, Berkeley, CA, USA) was employed for data analysis.

\section{Results}

\section{Amount of water applied and WUE}

Throughout each experiment, thirty irrigation actions; a total of 30.6,24.5, and $18.4 \mathrm{~L}$ of water per plant were applied to 100,80 , and $60 \%$ of FC irrigation treatments, respectively. Therefore, 80 and $60 \%$ of FC irrigation treatments saved approximately 19.9 and $39.9 \%$ of water, respectively compared to the $100 \%$ of FC irrigation treatment.

For irrigation regimes, WUE (\%) increased significantly (by 98.2\%) with a decrease in water supply by $20 \%$, while significantly decreased (by $19.7 \%$ ) with a water supply decrease by $40 \%$ of FC compared to full irrigated plants (Table 1).

Regarding GSH treatments, GSH significantly increased WUE (by 98.8\%) when applied at $0.4 \mathrm{mM}$, and further increased WUE (by 237.1\%) when applied at $0.8 \mathrm{mM}$ compared to the control (foliar spray with water) (Table 1).

The interaction effect between irrigation treatments and GSH applications was significant for WUE (Figure 1). The highest WUE (215.4\%) was obtained from the interactive treatment of the irrigation regime of $80 \%$ of FC $\times$ GSH at $0.8 \mathrm{mM}$ followed by the irrigation regime of $80 \%$ of FC $\times \mathrm{GSH}$ at $0.4 \mathrm{mM}$ (WUE $=$ $130 \%$ ), then the irrigation regime of $100 \%$ of FC $\times \mathrm{GSH}$ at $0.8 \mathrm{mM}$ (WUE $=110 \%$ ), while the lowest WUE (30.4 or $30.3 \%$ ) was obtained from the interactive treatments of the irrigation regimes of 100 or $60 \%$ of FC $\times$ GSH at $0 \mathrm{mM}$ (water), respectively.

\section{Growth parameters and fruit yield components}

For irrigation treatments, chili pepper growth parameters (e.g., No. of branches plant-1, No. of leaves plant -1 , fresh and dry weights of shoot and root systems), and fruit yield components (e.g., No. of fruits plant1 , fresh and dry weights of fruits plant-1) were significantly decreased with a decrease in irrigation water by $20 \%$ (i.e., $80 \%$ of soil field capacity; FC) or by $40 \%$ (i.e., $60 \%$ of FC) compared to the control (irrigation at 
$100 \%$ of FC) (Table 1). Irrigation water provided at 80 or $60 \%$ of FC decreased plant height by 21.5 or $43.8 \%$, No. of branches plant -1 by 18.6 or $40.4 \%$, No. of leaves plant ${ }^{-1}$ by 18.2 or $39.3 \%$, fresh weight of plant shoot by 18.0 or $35.5 \%$, fresh weight of plant root by 26.6 or $48.3 \%$, dry weight of plant shoot by 25.1 or $46.4 \%$, dry weight of plant root by 22.9 or $44.3 \%$, respectively, compared to the control. Regarding yield components, while irrigation by $80 \%$ of FC increased No. of fruits plant ${ }^{-1}$, fresh weight of fruits plant-1, and dry weight of fruits plant ${ }^{-1}$ by $58.9,58.9$, and $71.6 \%$, respectively, irrigation by $60 \%$ of FC decreased these yield parameters by $52.4,51.7$, and $46.6 \%$, respectively compared to the control.

Concerning glutathione (GSH) foliar treatments, the above growth and fruit yield parameters of the chili pepper plant increased significantly with a foliar spray of $0.4 \mathrm{mM} \mathrm{GSH}$, and further increased with 0.8 $\mathrm{mM}$ GSH compared to the control (foliar spray with water) (Table 1). GSH leafy-provided at 0.4 or $0.8 \mathrm{mM}$ increased plant height by 44.3 or $81.6 \%$, No. of branches plant ${ }^{-1}$ by 39.6 or $63.7 \%$, No. of leaves plant -1 by 21.3 or $37.8 \%$, fresh weight of plant shoot by 27.5 or $60.3 \%$, fresh weight of plant root by 41.0 or $94.0 \%$, dry weight of plant shoot by 35.4 or $66.4 \%$, dry weight of plant root by 41.6 or $96.6 \%$, No. of fruits plant -1 by 98.3 or $235.0 \%$, plant fresh fruits by 102.0 or $241.0 \%$, and plant dry fruits by 109.3 or $258.1 \%$, respectively, compared to the control.

The interaction effect between irrigation treatments and GSH applications was significant for chili pepper plant growth and yield parameters (Figure 1). In general, the highest growth parameters were obtained from the interactive treatment of the irrigation regime of 80 or $100 \%$ of FC $\times \mathrm{GSH}$ at $0.8 \mathrm{mM}$ followed by the irrigation regime of 80 or $100 \%$ of FC $\times \mathrm{GSH}$ at $0.4 \mathrm{mM}$, while the lowest growth parameters were obtained from the interactive treatments of the irrigation regime of $60 \%$ of $\mathrm{FC} \times \mathrm{GSH}$ at $0 \mathrm{mM}$ (water).
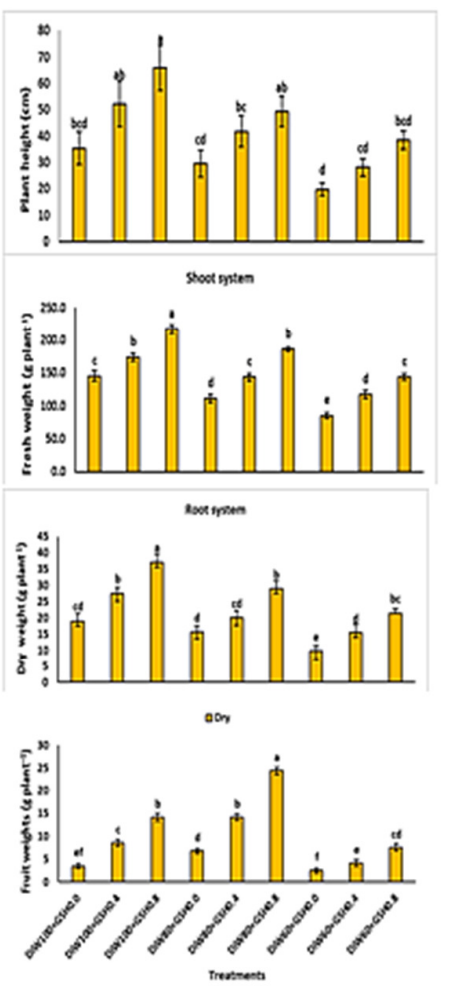
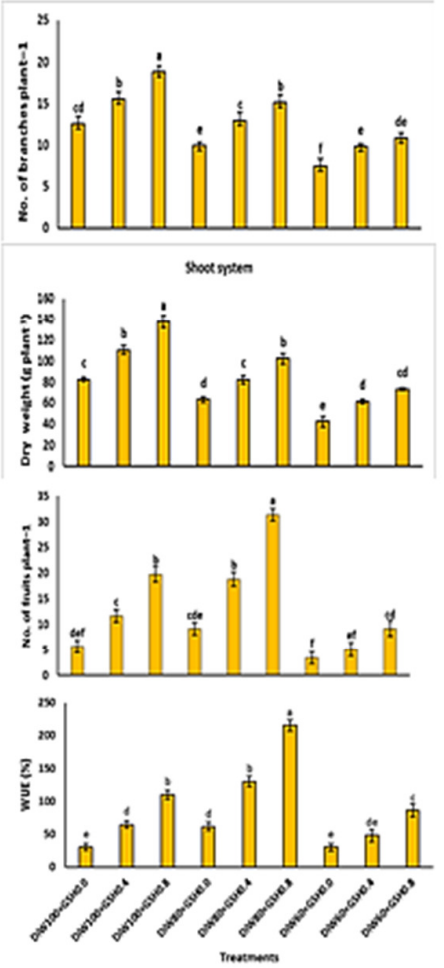
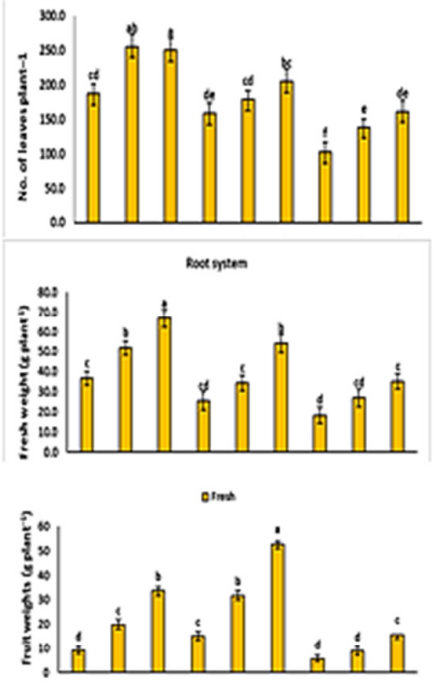

Figure 1. The effect of the interaction between deficiency of irrigation water (DiW) and spraying of glutathione (GSH) on growth characteristics and fruit yield components of chili pepper plants grown in saline soil $\left(\mathrm{EC}=6.74 \mathrm{dS} \mathrm{m^{-1 } )}\right.$ 
Table 1. The effect of deficiency of irrigation water (DiW) and spraying of glutathione (GSH) on growth characteristics and fruit yield components of chili pepper plants grown in saline soil $\left(\mathrm{EC}=6.74 \mathrm{dS} \mathrm{m}^{-1}\right)$

\begin{tabular}{|c|c|c|c|c|c|c|c|c|}
\hline \multirow[b]{2}{*}{ Treatments } & \multirow{2}{*}{$\begin{array}{l}\text { Plant } \\
\text { height } \\
(\mathrm{cm})\end{array}$} & \multirow{2}{*}{$\begin{array}{c}\text { No. of } \\
\text { branches } \\
\text { plant }^{-1}\end{array}$} & \multirow{2}{*}{$\begin{array}{c}\text { No. of leaves } \\
\text { plant }^{-1}\end{array}$} & \multicolumn{3}{|c|}{ Fresh weight $(\mathrm{g})$} & \multicolumn{2}{|c|}{ Dry weight $(\mathrm{g})$} \\
\hline & & & & \multicolumn{2}{|c|}{$\begin{array}{l}\text { Shoot } \\
\text { system }\end{array}$} & $\begin{array}{c}\text { Root } \\
\text { system }\end{array}$ & $\begin{array}{l}\text { Shoot } \\
\text { system }\end{array}$ & $\begin{array}{c}\text { Root } \\
\text { system }\end{array}$ \\
\hline $\mathrm{DiW}(\mathrm{FC} \%)$ & ${ }^{*}$ & $*$ & * & \multicolumn{2}{|c|}{$*$} & $*$ & $*$ & $*$ \\
\hline 100 & $51.2 \pm 7.8 \mathrm{a}$ & $15.6 \pm 0.8 \mathrm{a}$ & $221.1 \pm 17.2 \mathrm{a}$ & \multicolumn{2}{|c|}{$178.7 \pm 7.0 \mathrm{a}$} & $51.8 \pm 3.9 \mathrm{a}$ & $110.4 \pm 4.1 \mathrm{a}$ & $28.0 \pm 2.1 \mathrm{a}$ \\
\hline 80 & $40.2 \pm 5.6 b$ & $12.7 \pm 0.7 \mathrm{~b}$ & $180.8 \pm 15.8 b$ & \multicolumn{2}{|c|}{$146.6 \pm 4.8 b$} & $38.0 \pm 4.4 \mathrm{~b}$ & $82.7 \pm 3.9 \mathrm{~b}$ & $21.6 \pm 2.1 b$ \\
\hline 60 & $28.8 \pm 3.0 \mathrm{c}$ & $9.3 \pm 0.6 c$ & $134.3 \pm 14.0 \mathrm{c}$ & \multicolumn{2}{|c|}{$115.2 \pm 5.2 c$} & $26.8 \pm 4.1 \mathrm{c}$ & $59.2 \pm 2.8 \mathrm{c}$ & $15.6 \pm 1.9 \mathrm{c}$ \\
\hline $\mathrm{GSH}(\mathrm{mM})$ & ${ }^{*}$ & ${ }^{*}$ & ${ }^{*}$ & \multicolumn{2}{|c|}{$*$} & ${ }^{*}$ & $*$ & ${ }^{*}$ \\
\hline 0.0 (Control) & $28.2 \pm 4.5 c$ & $09.1 \pm 0.7 \mathrm{c}$ & $149.3 \pm 14.8 \mathrm{c}$ & \multicolumn{2}{|c|}{$113.6 \pm 6.0 \mathrm{c}$} & $26.8 \pm 4.0 \mathrm{c}$ & $62.8 \pm 3.5 \mathrm{c}$ & $14.9 \pm 1.9 \mathrm{c}$ \\
\hline 0.4 & $40.7 \pm 5.9 b$ & $12.7 \pm 0.7 \mathrm{~b}$ & $181.1 \pm 14.6 \mathrm{~b}$ & \multicolumn{2}{|c|}{$144.8 \pm 5.8 \mathrm{~b}$} & $37.8 \pm 4.0 \mathrm{~b}$ & $85.0 \pm 3.4 \mathrm{~b}$ & $21.1 \pm 2.2 \mathrm{~b}$ \\
\hline 0.8 & $51.2 \pm 6.0 \mathrm{a}$ & $14.9 \pm 0.7 \mathrm{a}$ & $205.7 \pm 17.8 \mathrm{a}$ & \multicolumn{2}{|c|}{$182.1 \pm 5.2 \mathrm{a}$} & $52.0 \pm 4.4 \mathrm{a}$ & $104.5 \pm 3.9 \mathrm{a}$ & $29.3 \pm 2.1 \mathrm{a}$ \\
\hline $\mathrm{DiW} \times \mathrm{GSH}$ & $*$ & $*$ & $*$ & \multicolumn{2}{|c|}{$*$} & $*$ & $*$ & $*$ \\
\hline Treatments & \multicolumn{2}{|c|}{ No. of fruits plant ${ }^{-1}$} & \multicolumn{2}{|c|}{$\begin{array}{l}\text { Fresh fruit weights } \\
\quad\left(\text { g plant }^{-1}\right)\end{array}$} & \multicolumn{2}{|c|}{$\begin{array}{c}\text { Dry fruit weights } \\
\left(\mathrm{g} \mathrm{plant}^{-1}\right)\end{array}$} & \multicolumn{2}{|c|}{$\begin{array}{c}\text { WUE } \\
(\%)\end{array}$} \\
\hline $\mathrm{DiW}(\mathrm{FC} \%)$ & \multicolumn{2}{|c|}{ * } & \multicolumn{2}{|c|}{$*$} & \multicolumn{2}{|c|}{$*$} & \multicolumn{2}{|c|}{$*$} \\
\hline 100 & \multicolumn{2}{|c|}{$12.4 \pm 1.3 \mathrm{~b}$} & \multicolumn{2}{|c|}{$20.9 \pm 1.8 b$} & \multicolumn{2}{|c|}{$8.8 \pm 0.7 b$} & \multicolumn{2}{|c|}{$68.4 \pm 5.7 b$} \\
\hline 80 & \multicolumn{2}{|c|}{$19.7 \pm 1.3 \mathrm{a}$} & \multicolumn{2}{|l|}{$33.2 \pm 1.9 \mathrm{a}$} & \multicolumn{2}{|c|}{$15.1 \pm 0.7 \mathrm{a}$} & \multicolumn{2}{|c|}{$135.6 \pm 7.6 \mathrm{a}$} \\
\hline 60 & \multicolumn{2}{|c|}{$5.9 \pm 1.3 \mathrm{c}$} & \multicolumn{2}{|l|}{$10.1 \pm 1.5 \mathrm{c}$} & & $4.7 \pm 0.7 \mathrm{c}$ & 54.9 & $8.1 \mathrm{c}$ \\
\hline $\mathrm{GSH}(\mathrm{mM})$ & * & & $*$ & & & $*$ & & \\
\hline 0.0 (Control) & $6.0 \pm$ & $1.2 \mathrm{c}$ & $10.0 \pm 1.4 \mathrm{c}$ & & & $3 \pm 0.5 \mathrm{c}$ & 40.7 & $5.7 \mathrm{c}$ \\
\hline 0.4 & $11.9 \pm$ & $1.3 \mathrm{~b}$ & $20.2 \pm 1.8 \mathrm{~b}$ & & & $.0 \pm 0.7 b$ & 80.9 & $7.5 \mathrm{~b}$ \\
\hline 0.8 & $20.1 \pm$ & $1.4 \mathrm{a}$ & $34.1 \pm 2.0 \mathrm{a}$ & & & $5.4 \pm 0.8 \mathrm{a}$ & 137.2 & $=8.3 \mathrm{a}$ \\
\hline $\mathrm{DiW} \times \mathrm{GSH}$ & * & & $*$ & & & $*$ & 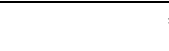 & \\
\hline
\end{tabular}

\section{Total chlorophyll, some osmoprotectant, and antioxidant contents}

Regarding irrigation treatments, deficit irrigation water treatments did not affect the soluble sugars content, but significantly decreased the chlorophyll content (measured as a SPAD index), while they significantly increased the contents of proline, phenols, capsaicin, ascorbic acid (AsA), and glutathione (GSH) compared to full irrigation treatment (Table 2). Irrigation water provided at 80 or $60 \%$ of FC decreased SPAD index by 11.5 or $20.6 \%$, while increased proline content by 18.9 or $28.9 \%$, phenolic content by 46.5 or $55.8 \%$, capsaicin content by 9.0 or $19.1 \%$, AsA content by 48.1 or $105.9 \%$, and GSH content by 64.6 or $149.0 \%$ compared to the control.

As for GSH foliar treatments, 0.4 or $0.8 \mathrm{mM}$ GSH did not affect the proline content, while they significantly increased the contents of SPAD index, proline, phenols, capsaicin, ascorbic acid (AsA), and glutathione (GSH) compared to the control (foliar spray with water) (Table 2). GSH foliar-sprayed at 0.4 or $0.8 \mathrm{mM}$ increased SPAD index by 4.4 or $11.1 \%$, soluble sugars content by 14.7 or $30.2 \%$, phenolic content by 7.7 or $25.0 \%$, capsaicin content by 14.0 or $25.6 \%$, AsA content by 8.0 or $18.1 \%$, and GSH by 26.4 or $55.0 \%$ compared to the control.

The interaction effect between irrigation treatments and GSH applications was significant for the SPAD index and all tested osmoprotectant and antioxidant parameters (Figure 2). In general, the highest values of osmoprotectant and antioxidant parameters were obtained from the interactive treatment of the irrigation 
regime of $60 \%$ of FC $\times$ GSH at $0.8 \mathrm{mM}$ followed by the irrigation regime of $60 \%$ of FC $\times \mathrm{GSH}$ at $0.4 \mathrm{mM}$, then the irrigation regime of $80 \%$ of $\mathrm{FC} \times \mathrm{GSH}$ at $0.8 \mathrm{mM}$.
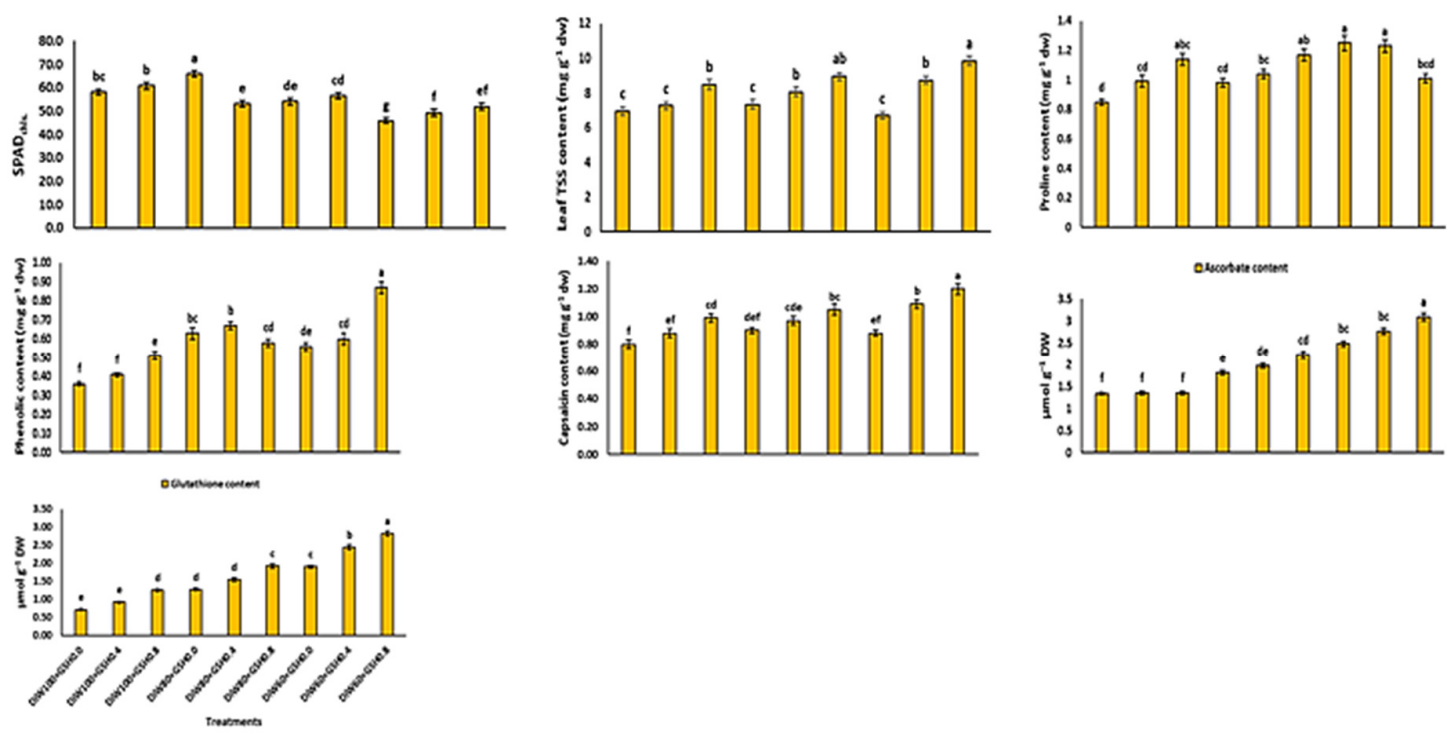

Figure 2. The effect of the interaction between deficiency of irrigation water (DiW) and spraying of glutathione $(\mathrm{GSH})$ on SPADchls., some osmoprotectant and antioxidant contents in dry leaves of chili pepper plants grown in saline soil $\left(\mathrm{EC}=6.74 \mathrm{dS} \mathrm{\textrm {m } ^ { - 1 } )}\right.$

Table 2. The effect of deficiency of irrigation water (DiW) and spraying of glutathione (GSH) on $\mathrm{SPAD}_{\text {chls. }}$, some osmoprotectant and antioxidant contents in dry leaves of chili pepper plants grown in saline soil $\left(\mathrm{EC}=6.74 \mathrm{dS} \mathrm{m}^{-1}\right)$

\begin{tabular}{|c|c|c|c|c|c|c|c|}
\hline \multirow{2}{*}{ Treatments } & \multirow{2}{*}{$\begin{array}{c}\text { SPAD } \\
\text { chls. }\end{array}$} & $\begin{array}{c}\text { Leaf TSS } \\
\text { content }\end{array}$ & $\begin{array}{c}\text { Leaf } \\
\text { Proline } \\
\text { content }\end{array}$ & $\begin{array}{c}\text { Leaf } \\
\text { Phenolic } \\
\text { content }\end{array}$ & $\begin{array}{c}\text { Fruit } \\
\text { Capsaicin } \\
\text { content }\end{array}$ & $\begin{array}{c}\text { Leaf } \\
\text { Ascorbate } \\
\text { content }\end{array}$ & $\begin{array}{c}\text { Leaf } \\
\text { Glutathione } \\
\text { content }\end{array}$ \\
\cline { 3 - 8 } & & \multicolumn{7}{|c|}{$\mathrm{mg} \mathrm{g}^{-1} \mathrm{DW}$} \\
\hline DiW (FC\%) & $*$ & $\mathrm{~ns}$ & $*$ & ${ }^{*}$ & $\mathrm{mmol} \mathrm{g}^{-1} \mathrm{DW}$ \\
\hline 100 & $61.7 \pm 1.4 \mathrm{a}$ & $7.56 \pm 0.26$ & $0.90 \pm 0.03 \mathrm{c}$ & $0.43 \pm 0.01 \mathrm{c}$ & $0.89 \pm 0.03 \mathrm{c}$ & $1.35 \pm 0.03 \mathrm{c}$ & $0.96 \pm 0.03 \mathrm{c}$ \\
\hline 80 & $54.6 \pm 1.4 \mathrm{~b}$ & $8.12 \pm 0.25$ & $1.07 \pm 0.03$ & $0.63 \pm 0.02 \mathrm{~b}$ & $0.97 \pm 0.03 \mathrm{~b}$ & $2.00 \pm 0.06$ & $1.58 \pm 0.05 \mathrm{~b}$ \\
\hline 60 & $49.0 \pm 1.3 \mathrm{c}$ & $8.43 \pm 0.23$ & $1.16 \pm 0.04 \mathrm{a}$ & $0.67 \pm 0.03 \mathrm{a}$ & $1.06 \pm 0.03 \mathrm{a}$ & $2.78 \pm 0.07 \mathrm{a}$ & $2.39 \pm 0.06 \mathrm{a}$ \\
\hline $\mathrm{GSH}(\mathrm{mM})$ & $*$ & $*$ & $\mathrm{~ns}$ & $*$ & $*$ & $*$ & $*$ \\
\hline $0.0(\mathrm{Control})$ & $52.4 \pm 1.3 \mathrm{c}$ & $6.99 \pm 0.23 \mathrm{c}$ & $1.03 \pm 0.03$ & $0.52 \pm 0.02 \mathrm{c}$ & $0.86 \pm 0.02 \mathrm{c}$ & $1.88 \pm 0.05 \mathrm{c}$ & $1.29 \pm 0.03 \mathrm{c}$ \\
\hline 0.4 & $54.7 \pm 1.5 \mathrm{~b}$ & $8.02 \pm 0.24 \mathrm{~b}$ & $1.09 \pm 0.04$ & $0.56 \pm 0.02 \mathrm{~b}$ & $0.98 \pm 0.03 \mathrm{~b}$ & $2.03 \pm 0.05$ & $1.63 \pm 0.05 \mathrm{~b}$ \\
\hline 0.8 & $58.2 \pm 1.4 \mathrm{a}$ & $9.10 \pm 0.26 \mathrm{a}$ & $1.11 \pm 0.04$ & $0.65 \pm 0.02 \mathrm{a}$ & $1.08 \pm 0.04 \mathrm{a}$ & $2.22 \pm 0.06 \mathrm{a}$ & $2.00 \pm 0.05 \mathrm{a}$ \\
\hline DiW $\times$ GSH & $*$ & $*$ & $*$ & $*$ & $*$ & $*$ & $*$ \\
\hline
\end{tabular}

${ }^{* *}$ and ${ }^{*}$ indicate, respectively, differences at $P \leq 0.01$ and $P \leq 0.05$ probability level, and "ns" indicates not a significant difference. Means $( \pm$ SE) followed by the same letter in each column are not significantly different according to the LSD test $(P \leq 0.01$ and $P \leq 0.05)$. EC $=$ electrical conductivity, $\mathrm{FC}=$ field capacity, $\mathrm{mM}=$ millimole, Chls= chlorophylls, and TSS $=$ total soluble sugars.

\section{Leaf macro-and micro-nutrients status, $\mathrm{Na}^{+}$and $\mathrm{Cl}$ contents, and $\mathrm{K}^{+} / \mathrm{Na}^{+}$ratio}

As for irrigation treatments, leaf macro-and micro-nutrients contents (e.g., N, P, K, Ca, Mg, Fe, Zn, and $\mathrm{Mn})$ and $\mathrm{K}^{+} / \mathrm{Na}^{+}$ratio were significantly decreased, while the contents of $\mathrm{Na}^{+}$and $\mathrm{Cl}^{-}$were significantly increased with a decrease in irrigation water by $20 \%$ (i.e., $80 \%$ of FC) or by $40 \%$ (i.e., $60 \%$ of FC) compared to 
full irrigation ( $100 \%$ of FC) (Table 3). Irrigation water provided at 80 or $60 \%$ of FC decreased N content by 10.7 or $15.7 \%$, P content by 16.1 or $32.3 \%$, K content by 13.2 or $32.9 \%$, Ca content by 6.4 or $24.8 \%, \mathrm{Mg}$ content by 7.3 or $13.5 \%$, Fe content by 8.0 or $17.7 \%$, Zn content by 17.1 or $26.8 \%$, Mn content by 7.7 or $20.5 \%$, and $\mathrm{K}^{+} / \mathrm{Na}^{+}$ratio by 18.5 or $40.2 \%$, while increased $\mathrm{Na}^{+}$content by 9.1 or $13.6 \%$, and $\mathrm{Cl}^{-}$content by 9.5 or $25.5 \%$, respectively, compared to the control.

Regarding GSH foliar treatments, the above leaf macro- and micro-nutrients contents and $\mathrm{K}^{+} / \mathrm{Na}^{+}$ratio were significantly increased, while $\mathrm{Na}^{+}$and $\mathrm{Cl}^{-}$contents were significantly decreased with foliar spray of 0.4 or $0.8 \mathrm{mM}$ GSH compared to the control (foliar spray with water) (Table 3). GSH leafy-provided at 0.4 or 0.8 $\mathrm{mM}$ increased $\mathrm{N}$ content by 21.6 or $37.2 \%$, P content by 85.7 or $171.4 \%, \mathrm{~K}^{+}$content by 18.9 or $38.2 \%$, Ca content by 13.6 or $27.0 \%$, Mg content by 0.6 or $3.4 \%$, Fe content by 12.0 or $26.6 \%$, Zn content by 280.0 or $470.0 \%$, Mn content by 171.4 or $285.7 \%$, and $\mathrm{K}^{+} / \mathrm{Na}^{+}$ratio by 29.8 or $71.1 \%$, while decreased $\mathrm{Na}^{+}$content by 7.7 or $19.2 \%$, and $\mathrm{Cl}^{-}$content by 9.9 or $23.3 \%$, respectively, compared to the control.

The interaction effect between irrigation treatments and GSH applications was significant for leaf macro- and micro-nutrient status, $\mathrm{Na}^{+}$and $\mathrm{Cl}^{-}$contents, and $\mathrm{K}^{+} / \mathrm{Na}^{+}$ratio of the chili pepper plant (Fig. 3). Generally, the highest nutrient contents and $\mathrm{K}^{+} / \mathrm{Na}^{+}$ratio, and the lowest $\mathrm{Na}^{+}$and $\mathrm{Cl}^{-}$contents were obtained from the interactive treatment of the irrigation regime of $80 \%$ of FC $\times \mathrm{GSH}$ at $0.8 \mathrm{mM}$ followed by the irrigation regime of $100 \%$ of FC $\times \mathrm{GSH}$ at $0.8 \mathrm{mM}$, then the irrigation regime of $100 \%$ of $\mathrm{FC} \times \mathrm{GSH}$ at 0.4 $\mathrm{mM}$.

Table 3. The effect of deficiency of irrigation water (DiW) and spraying of glutathione (GSH) on macroand micro-nutrients, $\mathrm{Na}^{+}$, and $\mathrm{Cl}^{-}$contents, as well as $\mathrm{K}^{+} / \mathrm{Na}^{+}$ratio in dry leaves of chili pepper plants grown in saline soil $\left(\mathrm{EC}=6.74 \mathrm{dS} \mathrm{m}^{-1}\right)$

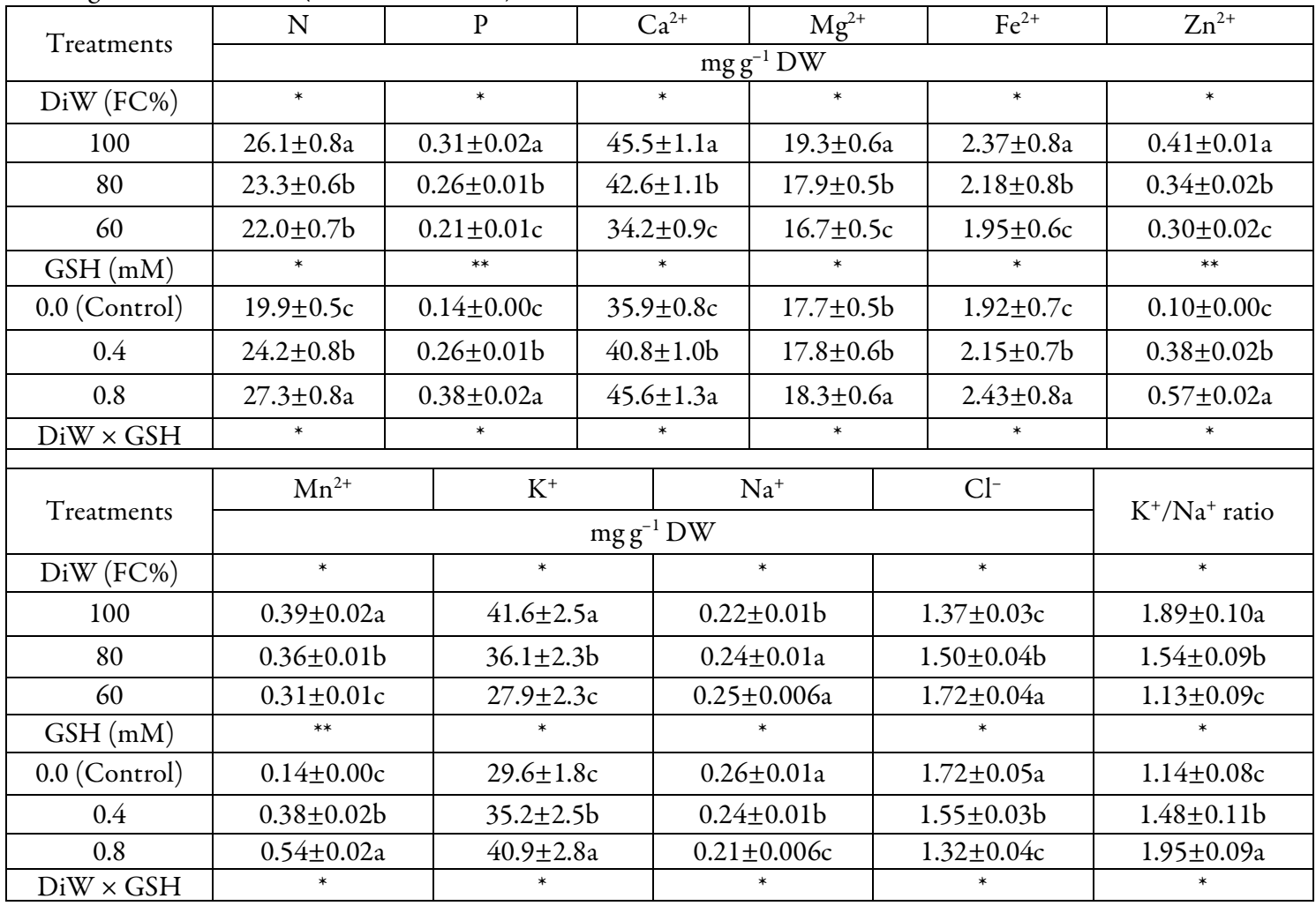

${ }^{* *}$ and ${ }^{*}$ indicate, respectively, differences at $P \leq 0.01$ and $P \leq 0.05$ probability level, and "ns" indicates not a significant difference. Means $( \pm$ SE) followed by the same letter in each column are not significantly different according to the LSD test $(P \leq 0.01$ and $P \leq 0.05)$. EC = electrical conductivity, $\mathrm{FC}=$ field capacity, $\mathrm{mM}=$ millimole, $\mathrm{DW}=$ dry weight, $\mathrm{N}=$ nitrogen, $\mathrm{P}=$ phosphorus, $\mathrm{Ca}=$ calcium, $\mathrm{Mg}=$ magnesium, $\mathrm{Fe}=$ iron, $\mathrm{Zn}=$ zinc, $\mathrm{Mn}=$ manganese, $\mathrm{K}=$ potassium, $\mathrm{Na}=$ sodium, and $\mathrm{Cl}=$ chlorine . 

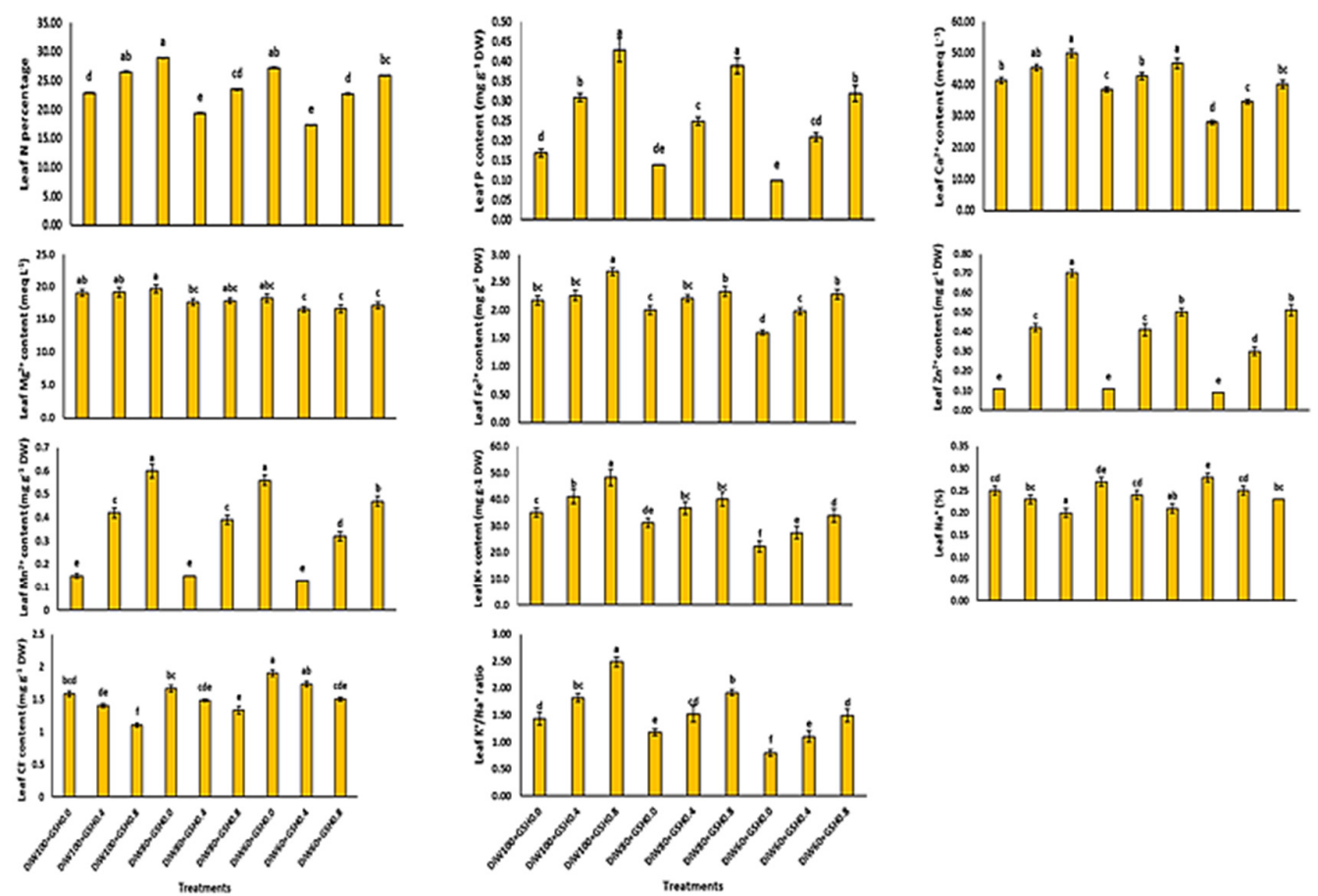

Figure 3. The effect of the interaction between deficiency of irrigation water (DiW) and spraying of glutathione (GSH) on macro-and micro-nutrients, $\mathrm{Na}^{+}$, and $\mathrm{Cl}^{-}$contents, as well as $\mathrm{K}^{+} / \mathrm{Na}^{+}$ratio in dry leaves of chili pepper plants grown in saline soil $\left(\mathrm{EC}=6.74 \mathrm{dS} \mathrm{m} \mathrm{m}^{-1}\right)$

\section{Leaf and stem anatomy}

As for irrigation treatments, the reduction in irrigation water by 20 or $40 \%$ (i.e., 80 or $60 \%$ of FC, respectively) resulted in a marked decrease in all features of leaf and stem anatomy of chili pepper plant compared to full irrigation treatment (Table $4 \mathrm{a}, \mathrm{b}$ ). Declines in leaf and stem anatomy features were more pronounced under deficit irrigation at $60 \%$ of FC than deficit irrigation at $80 \%$ of FC.

Concerning GSH foliar treatments, 0.4 or $0.8 \mathrm{mM} \mathrm{GSH}$ significantly recovered all features of leaf and stem anatomy compared to the control (foliar spray with water) (Table $4 \mathrm{a}, \mathrm{b}$ ). Improvements in leaf and stem anatomy features were more noticeable with the application of GSH at $0.8 \mathrm{mM}$ compared to $0.4 \mathrm{mM}$.

The interaction effect between irrigation treatments and GSH applications was significant for the tested features of leaf and stem anatomy (Table $4 \mathrm{a}, \mathrm{b}$ ). Generally, the highest values of osmoprotectant and antioxidant parameters were obtained from the interactive treatment of the irrigation regime of $100 \%$ of $\mathrm{FC} \times$ GSH at $0.8 \mathrm{mM}$ followed by the irrigation regime of $80 \%$ of FC $\times \mathrm{GSH}$ at $0.8 \mathrm{mM}$, while the lowest growth parameters were obtained from the interactive treatments of the irrigation regime of $60 \%$ of FC $\times \mathrm{GSH}$ at 0 $\mathrm{mM}$ (water). 
Table 4a. The effect of deficiency of irrigation water (DiW) and spraying of glutathione (GSH) on leaf of chili pepper plants grown in saline soil $\left(\mathrm{EC}=6.74 \mathrm{dS} \mathrm{m}^{-1}\right)$

\begin{tabular}{|c|c|c|c|c|c|c|c|c|}
\hline \multirow{3}{*}{ Treatments } & \multicolumn{8}{|c|}{ Leaf } \\
\hline & \multicolumn{2}{|c|}{ Midvien } & \multicolumn{2}{|c|}{ Median vb } & \multirow{2}{*}{$\begin{array}{c}\text { Average } \\
\text { Mx vessels } \\
\text { diameter } \\
(\mu \mathrm{m})\end{array}$} & \multirow[b]{2}{*}{$\begin{array}{l}\text { Blade thick. } \\
\qquad(\mu \mathrm{m})\end{array}$} & \multirow{2}{*}{$\begin{array}{c}\text { Palisade } \\
\text { thick. } \\
(\mu \mathrm{m})\end{array}$} & \multirow{2}{*}{$\begin{array}{c}\text { Spongy } \\
\text { thick. } \\
(\mu \mathrm{m})\end{array}$} \\
\hline & $\begin{array}{l}\text { Width } \\
(\mu \mathrm{m})\end{array}$ & $\begin{array}{c}\text { Length } \\
(\mu \mathrm{m})\end{array}$ & $\begin{array}{l}\text { Width } \\
(\mu \mathrm{m})\end{array}$ & $\begin{array}{c}\text { Length } \\
(\mu \mathrm{m})\end{array}$ & & & & \\
\hline $\mathrm{DiW}(\mathrm{FC} \%)$ & * & * & * & ns & $*$ & * & ns & * \\
\hline 100 & $767 \pm 67 a$ & $560 \pm 43 a$ & $490 \pm 42 a$ & $123 \pm 9$ & $12 \pm 1 \mathrm{a}$ & $213 \pm 17 a$ & $70 \pm 5$ & $113 \pm 9 a$ \\
\hline 80 & $653 \pm 57 b$ & $567 \pm 50 a$ & $383 \pm 37 b$ & $137 \pm 13$ & $10 \pm 1 b$ & $193 \pm 17 b$ & $70 \pm 6$ & $100 \pm 6 b$ \\
\hline 60 & $537 \pm 48 c$ & $507 \pm 43 b$ & $240 \pm 18 c$ & $123 \pm 10$ & $10 \pm 1 b$ & $203 \pm 22 \mathrm{ab}$ & $67 \pm 6$ & $100 \pm 7 b$ \\
\hline GSH (mM) & $*$ & $*$ & $*$ & $*$ & $*$ & $*$ & $*$ & $*$ \\
\hline 0.0 (Control) & $613 \pm 53 c$ & $533 \pm 43 b$ & $367 \pm 32 b$ & $133 \pm 11 \mathrm{a}$ & $10 \pm 1 b$ & $193 \pm 18 b$ & $70 \pm 7 a$ & $97 \pm 7 b$ \\
\hline 0.4 & $653 \pm 62 b$ & $510 \pm 40 b$ & $347 \pm 30 c$ & $107 \pm 8 b$ & $12 \pm 1 \mathrm{a}$ & $193 \pm 17 b$ & $63 \pm 5 b$ & $97 \pm 7 \mathrm{~b}$ \\
\hline 0.8 & $690 \pm 57 a$ & $590 \pm 53 a$ & $400 \pm 35 a$ & $143 \pm 12 a$ & $10 \pm 1 b$ & $223 \pm 20 a$ & $73 \pm 6 a$ & $120 \pm 8 a$ \\
\hline $\mathrm{DiW} \times \mathrm{GSH}$ & $*$ & $*$ & $*$ & $*$ & ${ }^{*}$ & $*$ & $*$ & $*$ \\
\hline $\mathrm{DiW}_{100} \times \mathrm{GSH}_{0.0}$ & $680 \pm 55 c$ & $500 \pm 35 c$ & $450 \pm 30 \mathrm{~b}$ & $130 \pm 11 \mathrm{ab}$ & $10 \pm 1 b$ & $180 \pm 15 c$ & $60 \pm 5 c$ & $100 \pm 8 b c$ \\
\hline $\mathrm{DiW}_{100} \times \mathrm{GSH}_{0.4}$ & $770 \pm 70 b$ & $530 \pm 40 c$ & $470 \pm 45 b$ & $100 \pm 8 c$ & $15 \pm 2 a$ & $220 \pm 15 \mathrm{ab}$ & $70 \pm 4 b$ & $110 \pm 10 b$ \\
\hline $\mathrm{DiW}_{100} \times \mathrm{GSH}_{0.8}$ & $850 \pm 75 a$ & $650 \pm 55 a$ & $550 \pm 50 a$ & $140 \pm 9 \mathrm{ab}$ & $10 \pm 1 b$ & $240 \pm 20 a$ & $80 \pm 6 a$ & $130 \pm 10 \mathrm{a}$ \\
\hline $\mathrm{DiW}_{80} \times \mathrm{GSH}_{0.0}$ & $640 \pm 55 c$ & $600 \pm 50 \mathrm{~b}$ & $400 \pm 45 c$ & $150 \pm 13 a$ & $10 \pm 1 b$ & $200 \pm 20 b c$ & $80 \pm 8 a$ & $100 \pm 7 \mathrm{bc}$ \\
\hline $\mathrm{DiW}_{80} \times \mathrm{GSH}_{0.4}$ & $650 \pm 60 c$ & $500 \pm 40 c$ & $350 \pm 30 \mathrm{~d}$ & $120 \pm 10 b c$ & $10 \pm 1 b$ & $180 \pm 15 c$ & $60 \pm 5 c$ & $100 \pm 6 b c$ \\
\hline $\mathrm{DiW}_{80} \times \mathrm{GSH}_{0.8}$ & $670 \pm 55 c$ & $600 \pm 60 b$ & $400 \pm 35 c$ & $140 \pm 15 \mathrm{ab}$ & $10 \pm 1 b$ & $200 \pm 15 b c$ & $70 \pm 6 b$ & $100 \pm 5 b c$ \\
\hline $\mathrm{DiW}_{60} \times \mathrm{GSH}_{0.0}$ & $520 \pm 50 \mathrm{~d}$ & $500 \pm 45 c$ & $250 \pm 20 \mathrm{e}$ & $120 \pm 10 \mathrm{bc}$ & $10 \pm 0 \mathrm{~b}$ & $200 \pm 20 b c$ & $70 \pm 7 b$ & $90 \pm 6 \mathrm{~cd}$ \\
\hline $\mathrm{DiW}_{60} \times \mathrm{GSH}_{0.4}$ & $540 \pm 55 d$ & $500 \pm 40 c$ & $220 \pm 15 e$ & $100 \pm 7 c$ & $10 \pm 1 b$ & $180 \pm 20 c$ & $60 \pm 5 c$ & $80 \pm 6 \mathrm{~d}$ \\
\hline $\mathrm{DiW}_{60} \times \mathrm{GSH}_{0.8}$ & $550 \pm 40 \mathrm{~d}$ & $520 \pm 45 c$ & $250 \pm 20 \mathrm{e}$ & $150 \pm 12 a$ & $10 \pm 1 b$ & $230 \pm 25 a$ & $70 \pm 5 b$ & $130 \pm 10 \mathrm{a}$ \\
\hline
\end{tabular}

${ }^{* *}$ and ${ }^{*}$ indicate, respectively, differences at $P \leq 0.01$ and $P \leq 0.05$ probability level, and "ns" indicates not a significant difference. Means $( \pm S E)$ followed by the same letter in each column are not significantly different according to the LSD test $(P \leq 0.01$ and $P \leq 0.05)$. EC= electrical conductivity, $\mathrm{FC}=$ field capacity, $\mathrm{mM}=$ millimole, $\mu \mathrm{m}=$ micrometer, $\mathrm{vb}=$ vascular bundle, $\mathrm{Mx}=$ metaxylem, thick $=$ thickness.

Table $4 \mathrm{~b}$. The effect of deficiency of irrigation water (DiW) and spraying of glutathione (GSH) on stem anatomic features of chili pepper plants grown in saline soil $\left(\mathrm{EC}=6.74 \mathrm{dS} \mathrm{m} \mathrm{m}^{-1}\right)$

\begin{tabular}{|c|c|c|c|c|c|c|c|}
\hline \multirow[b]{2}{*}{ Treatments } & \multicolumn{7}{|c|}{ Stem } \\
\hline & $\begin{array}{c}\text { Section } \\
\text { diameter }(\mu \mathrm{m})\end{array}$ & $\begin{array}{l}\text { Cortex } \\
\text { thick. } \\
(\mu \mathrm{m})\end{array}$ & $\begin{array}{c}\text { Cortical cell } \\
\text { No. }\end{array}$ & $\begin{array}{l}\text { Thick. of } \\
\text { vascular } \\
\text { cylinder } \\
(\mu \mathrm{m})\end{array}$ & $\begin{array}{c}\text { Diameter of } \\
\text { Mx vessels } \\
(\mu \mathrm{m})\end{array}$ & $\begin{array}{c}\text { Diameter of } \\
\text { pith cell } \\
(\mu \mathrm{m})\end{array}$ & $\begin{array}{c}\text { Diameter of } \\
\text { pith } \\
(\mu \mathrm{m})\end{array}$ \\
\hline $\mathrm{DiW}(\mathrm{FC} \%)$ & * & * & $\mathrm{ns}$ & $*$ & * & ns & * \\
\hline 100 & $4410 \pm 310 \mathrm{a}$ & $290 \pm 18 \mathrm{a}$ & $8.0 \pm 0.4$ & $1593 \pm 148 \mathrm{a}$ & $20 \pm 2 a$ & $47 \pm 4$ & $2307 \pm 180 \mathrm{a}$ \\
\hline 80 & $3383 \pm 272 b$ & $210 \pm 18 b$ & $7.7 \pm 0.5$ & $1160 \pm 117 \mathrm{~b}$ & $17 \pm 2 b$ & $43 \pm 4$ & $1733 \pm 152 b$ \\
\hline 60 & $2983 \pm 215 c$ & $193 \pm 16 c$ & $8.0 \pm 0.5$ & $1070 \pm 93 b$ & $17 \pm 2 b$ & $43 \pm 4$ & $1500 \pm 113 c$ \\
\hline $\mathrm{GSH}(\mathrm{mM})$ & ${ }^{*}$ & ${ }^{*}$ & $\mathrm{~ns}$ & $*$ & ${ }^{*}$ & ${ }^{*}$ & ${ }^{*}$ \\
\hline 0.0 (Control) & $3250 \pm 252 \mathrm{c}$ & $210 \pm 17 \mathrm{c}$ & $8.0 \pm 0.4$ & $1117 \pm 110 \mathrm{c}$ & $17 \pm 1 b$ & $40 \pm 3 b$ & $1707 \pm 145 \mathrm{c}$ \\
\hline 0.4 & $3650 \pm 260 \mathrm{~b}$ & $233 \pm 17 b$ & $8.0 \pm 0.5$ & $1293 \pm 117 \mathrm{~b}$ & $20 \pm 3 a$ & $47 \pm 3 a$ & $1833 \pm 138 \mathrm{~b}$ \\
\hline 0.8 & $3877 \pm 285 a$ & $250 \pm 18 \mathrm{a}$ & $8.0 \pm 0.5$ & $1413 \pm 126 a$ & $17 \pm 2 b$ & $47 \pm 5 a$ & $2000 \pm 162 \mathrm{a}$ \\
\hline $\mathrm{DiW} \times \mathrm{GSH}$ & $*$ & $*$ & $\mathrm{~ns}$ & $*$ & $*$ & * & * \\
\hline $\mathrm{DiW}_{100} \times \mathrm{GSH}_{0.0}$ & $3700 \pm 265 \mathrm{a}$ & $270 \pm 18 b$ & $8.0 \pm 0.5$ & $1250 \pm 130 \mathrm{~b}$ & $20 \pm 2 a$ & $40 \pm 3 c$ & $1870 \pm 145 \mathrm{c}$ \\
\hline $\mathrm{DiW}_{100} \times \mathrm{GSH}_{0.4}$ & $4650 \pm 315 a$ & $270 \pm 15 b$ & $7.7 \pm 0.4$ & $1700 \pm 155 \mathrm{a}$ & $20 \pm 3 a$ & $60 \pm 5 a$ & $2400 \pm 185 b$ \\
\hline $\mathrm{DiW}_{100} \times \mathrm{GSH}_{0.8}$ & $4880 \pm 350 \mathrm{a}$ & $330 \pm 21 \mathrm{a}$ & $8.3 \pm 0.4$ & $1830 \pm 160 \mathrm{a}$ & $20 \pm 2 a$ & $40 \pm 4 c$ & $2650 \pm 210 \mathrm{a}$ \\
\hline $\mathrm{DiW}_{80} \times \mathrm{GSH}_{0.0}$ & $3350 \pm 285 \mathrm{ab}$ & $190 \pm 20 \mathrm{~d}$ & $7.7 \pm 0.4$ & $1150 \pm 120 \mathrm{bc}$ & $15 \pm 1 b$ & $40 \pm 3 c$ & $1750 \pm 180 \mathrm{c}$ \\
\hline $\mathrm{DiW}_{80} \times \mathrm{GSH}_{0.4}$ & $3400 \pm 270 \mathrm{a}$ & $250 \pm 22 b c$ & $8.0 \pm 0.6$ & $1150 \pm 105 \mathrm{bc}$ & $20 \pm 2 a$ & $40 \pm 2 c$ & $1750 \pm 125 \mathrm{c}$ \\
\hline $\mathrm{DiW}_{80} \times \mathrm{GSH}_{0.8}$ & $3400 \pm 265 a$ & $190 \pm 14 d$ & $7.7 \pm 0.5$ & $1180 \pm 110 \mathrm{bc}$ & $15 \pm 2 b$ & $50 \pm 6 b$ & $1700 \pm 150 \mathrm{~cd}$ \\
\hline $\mathrm{DiW}_{60} \times \mathrm{GSH}_{0.0}$ & $2700 \pm 210 \mathrm{c}$ & $170 \pm 14 \mathrm{~d}$ & $8.0 \pm 0.4$ & $950 \pm 80 \mathrm{~d}$ & $15 \pm 1 b$ & $40 \pm 4 c$ & $1500 \pm 110 \mathrm{de}$ \\
\hline $\mathrm{DiW}_{60} \times \mathrm{GSH}_{0.4}$ & $2900 \pm 195 b c$ & $180 \pm 15 \mathrm{~d}$ & $8.0 \pm 0.6$ & $1030 \pm 90 \mathrm{~cd}$ & $20 \pm 3 a$ & $40 \pm 3 c$ & $1350 \pm 105 \mathrm{e}$ \\
\hline $\mathrm{DiW}_{60} \times \mathrm{GSH}_{0.8}$ & $3350 \pm 240 \mathrm{ab}$ & $230 \pm 18 c$ & $8.0 \pm 0.5$ & $1230 \pm 110 b$ & $15 \pm 2 b$ & $50 \pm 4 b$ & $1650 \pm 125 \mathrm{~cd}$ \\
\hline
\end{tabular}

${ }^{* *}$ and ${ }^{*}$ indicate, respectively, differences at $P \leq 0.01$ and $P \leq 0.05$ probability level, and "ns" indicates not a significant difference. Means $( \pm$ SE) followed by the same letter in each column are not significantly different according to the LSD test $(P \leq 0.01$ and $P \leq 0.05)$. EC = electrical conductivity, $\mathrm{FC}=$ field capacity, $\mathrm{mM}=$ millimole, $\mu \mathrm{m}=$ micrometer, $\mathrm{vb}=$ vascular bundle, $\mathrm{Mx}=$ metaxylem, thick= thickness. 

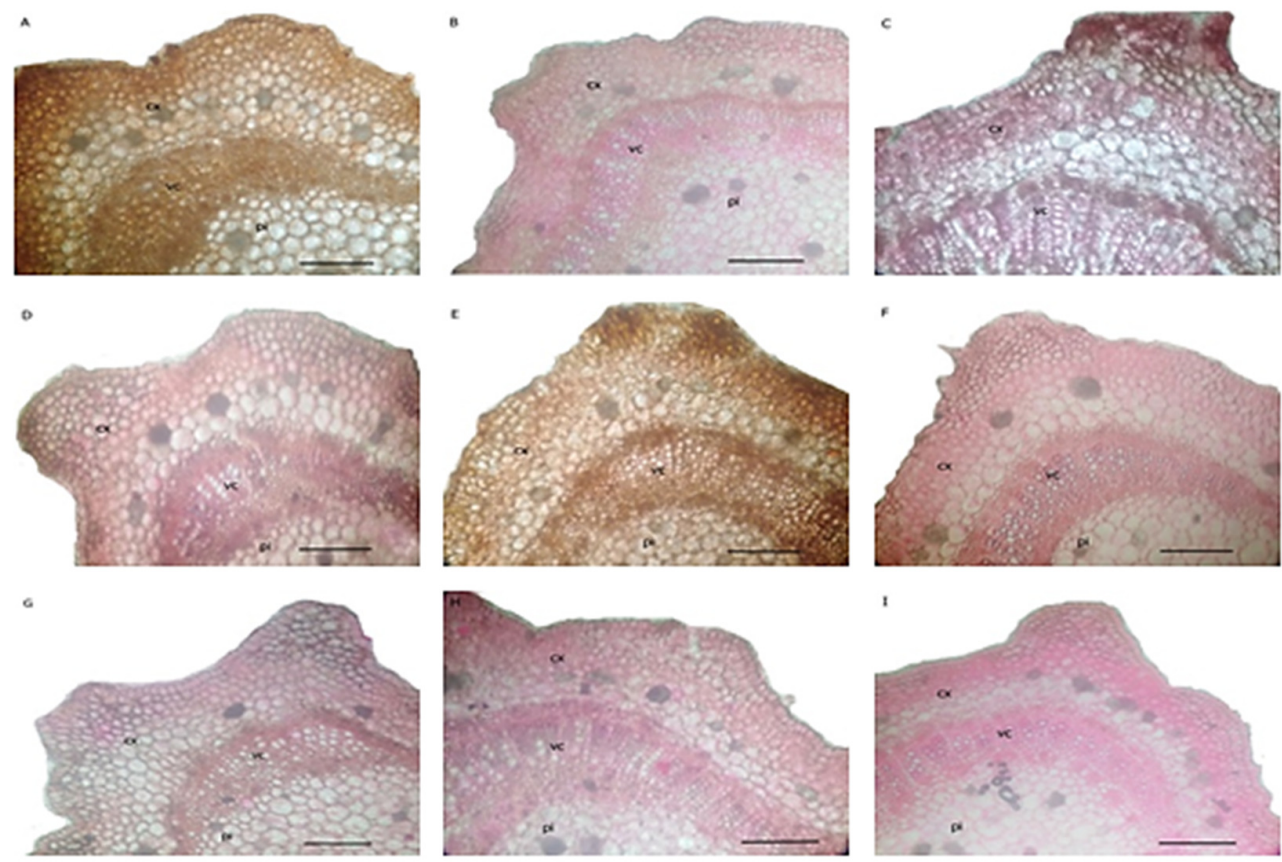

Figure 4. The effect of deficiency of irrigation water (DiW) and glutathione spraying (GSH) on stem anatomic features of chili pepper plants grown in saline soil $(\mathrm{cx}=$ cortex, $\mathrm{vc}=$ vascular cylinder, $\mathrm{pi}=$ pith. Line $=250 \mu \mathrm{m})$
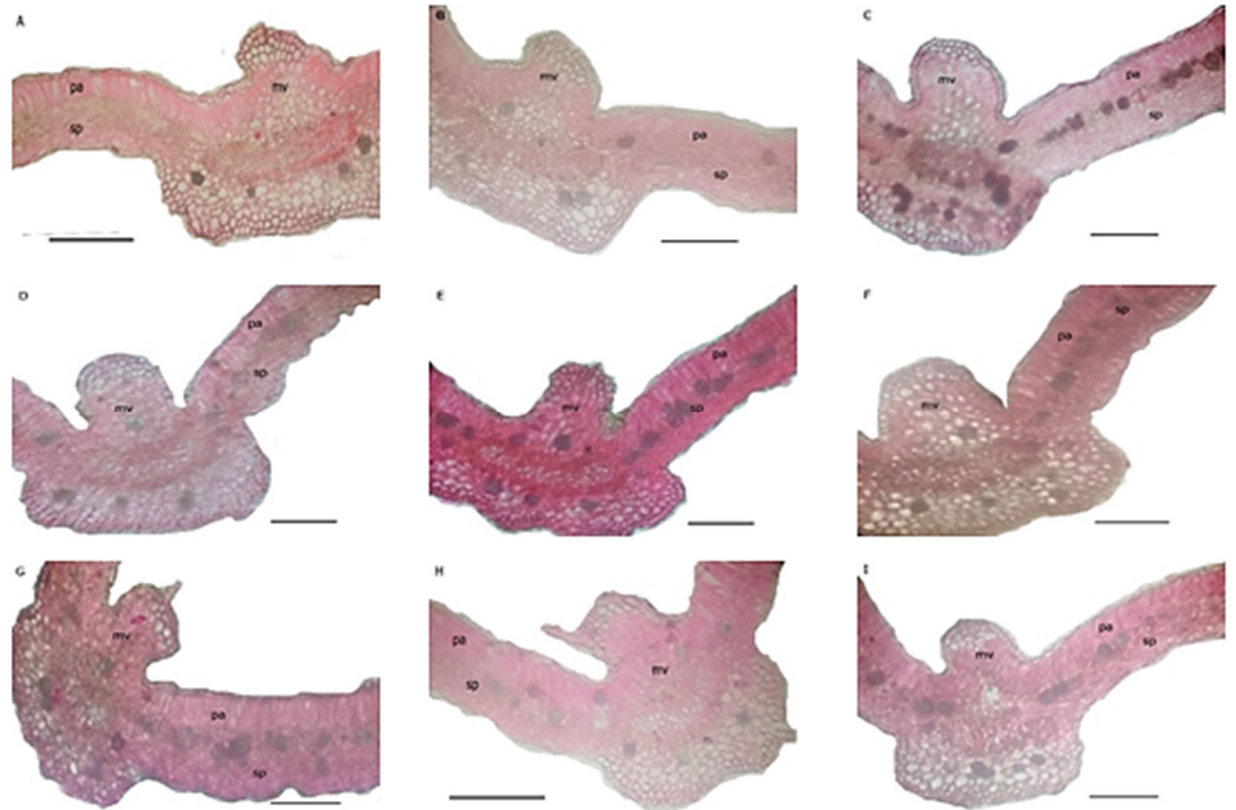

Figure 5. The effect of deficiency of irrigation water (DiW) and glutathione spraying (GSH) on leaf anatomic features of chili pepper plants grown in saline soil

$(\mathrm{mv}=$ midvein, $\mathrm{pa}=$ palisade tissue, $\mathrm{sp}=$ spongy tissue. Line $=250 \mu \mathrm{m})$. 


\section{Discussion}

Some researchers have described the importance of GSH in regulating plant physiological responses under adverse environmental conditions in different plant species (Cao et al., 2017; Sabetta et al., 2017; Sohag et al., 2020), but have not yet been studied in salt-stressed chili pepper plants grown under the stress of drought. In this study, GSH played a crucial role in reinforcing the tolerance to drought stress in chili pepper by regulating physiological and biochemical processes, including growth traits, chlorophylls, antioxidants, and regulation of ion influxes. The deficiency of irrigation water $(\mathrm{DiW})$ negatively affects the chili pepper growth criteria, fruit production, and developmental processes (Correia et al., 2001; Pinheiro and Chaves, 2011). These harmful effects are magnified under combined DiW + salt stress (Abd El-Mageed et al., 2018; Yang et al., 2019; Alharby et al., 2020).

Under saline soil conditions $\left(\mathrm{EC}=6.74 \mathrm{dS} \mathrm{m} \mathrm{m}^{-1}\right)$, in this study, a $40 \%$ decrease in required irrigation water (based on soil field capacity; FC) significantly reduced growth, especially fresh and dry weights, and fruit yield parameters of chili plants. On the other side, a $20 \%$ decrease in required irrigation water significantly increased fruit yield parameters despite lower growth criteria. Alharby et al. (2020) explained that plant growth and yield components are decreased under combined DiW + salt stress due to suppression in cellular expansion and division, chlorophyll content, and photosynthetic efficiency, although increases in endogenous antioxidants, phytohormones, and gene expression. Besides, reducing photosynthates partitioning in plants resulting from reducing leaf area, which leads to a decrease in dry matter and plant yields production (HongBo et al., 2008). However, the exogenous application of GSH restored the negatively affected growth and yield parameters that occurred due to the combined DiW + salt stress (Table 1; Figure 1). This growth and yield restoration by GSH may be attributed to its metabolic enzyme interactions with plant growth regulators, which are vital in the plant establishment scheme under normal conditions and stresses. Exogenous GSH causes higher levels of abscisic acid (ABA) to reduce the transpiration rate and regulates stomatal aperture and leaf water content, which may indicate a role in controlling leaf rolling (Chen et al., 2012). GSH is supposed to regulate growth-related functions, as raising endogenous GSH level enhances cell division in the root meristematic region (Vernoux et al., 2000), causing this region to be elongated, which is an important morphological adaptation to stress. Similar to our results, exogenous GSH enhanced plant growth and development grown under combined DiW + salt stress (Chen et al., 2012; Patil et al., 2014; El-Beltagi et al., 2020).

In this study, the highest fruit yield was observed with irrigation water supplementation at $80 \%$ of FC probably because this amount of water was sufficient to meet the water requirements of the crop during the flowering stage, fruit set, and fruit development to harvest even under soil salinity $\left(\mathrm{ECe}=6.74 \mathrm{dS} \mathrm{m} \mathrm{m}^{-1}\right)$. A similar result was obtained with Patil et al. (2014) who reported that deficit saline irrigation water had no adverse effect on bell pepper (Capsicum annuum) fruit yield. A 20\% decrease in the water supply (80\% of FC) to chili pepper plants saved water by more than $20 \%$ relative to the large increase in fruit yield and water use efficiency (WUE \%) compared to full irrigation (100\% of FC) (Table 1). Patil et al. (2014) also noted that $\mathrm{DiW}$ at $60 \%$ of ETc over the entire cropping period, which saved more water, led to a significantly higher WUE than all other DiW treatments. The increase in WUE by Ismail (2010) could be attributed to the increase in mass production per unit of water. In this study, the decrease in irrigation water by $20 \%$ not only increased WUE but also leafy-applied GSH significantly elevated it, besides the enhanced chili pepper growth and physiological performance under salt stress. GSH was applied to chili pepper leaves with significantly elevated endogenous levels of GSH and ascorbate (AsA) (Table 2) to enrich the GSH-AsA cycle, and thus as Foyer and Noctor (2005a) explained, it acts to eliminate ROS and maintain redox homeostasis in the tissues of stressed plants. The GSH-AsA cycle is a central component of the network of biochemical reactions that includes various antioxidants with the properties of redox reactions to efficiently eliminate ROS, thus prohibiting ROSmediated oxidative damage in stressed plant tissues (Foyer and Noctor, 2005a). Eventually, this leads to maintaining plant growth and satisfying yield. 
The chlorophyll content of plant leaves is one of the most important factors in determining the rate of photosynthesis and dry matter production (Dai et al., 2009). In this study, DiW applied to chili pepper plants damaged cell chloroplasts, which resulting in chlorophyll and photosynthesis impairment (Rady et al., 2020), associating with decreased plant growth (Table 1; Figure 1). Similar results indicated that combined DiW + salt stress minimized chlorophyll content (Abd El-Mageed et al., 2018; Alharby et al., 2020).

Several GSH mechanisms are involved in increasing WUE and mitigating salt stress and DiW damage. Under the DiW and soil salinity stresses in this study, soluble sugars content increased due to exogenous GSH, and proline level increased due to DiW elevated stress tolerance in chili pepper plants with increased WUE (Table 2; Figure 2). As compatible solutes, soluble sugars and proline play crucial roles in reducing stressinduced cell acidification and maintaining the osmoregulation as osmoprotectant substances, which also include phenolic compounds and the alkaloid capsaicin that were significantly increased under stresses (Table 2; Figure 2). These positive results combined with the increased endogenous GSH due to exogenous GSH markedly improved plant cell water status and maintained adequate tissue water content to improve metabolic processes under stress (Gill and Tuteja, 2010; Rady and Hemida, 2016; Zaki and Mohamed, 2018; Yang et al., 2019).

When antioxidants, including GSH, have many important roles as key mechanisms in mitigating the effects of abiotic stress on plants (Singh et al., 2016), the optimum GSH level applied to DiW-suffered chili plants can alleviate the adverse effects of $\mathrm{DiW}$ and reduce the impairment of chloroplasts (Hasanuzzaman et al., 2017). This result leads to increased chlorophyll contents in plant leaves even under DiW conditions, which are associated with excessive production of ROS (Rady et al., 2020). The photosynthesis process can be activated by applying GSH by regulating the PSII and/or the components of the plants' defense system against damage stimulated by excessive ROS production. The positive regulation of physiological and biochemical processes by GSH results in increased chlorophyll contents, and further activation of antioxidant machinery in DiW-suffered plants, which positively affect the efficiency of photosynthesis (Hasanuzzaman et al., 2017). The positive effect of GSH applied at $0.8 \mathrm{mM}$ explains the insignificant differences in the chlorophyll content (measured as a SPAD index) in the chili pepper plant leaves, which was obtained under both regular and DiW treatments (Table 2; Figure 2). GSH can prevent or delay the degradation of chlorophyll caused by droughtinduced oxidative stress by reducing biomarkers of oxidative stress and protecting the biosynthesis enzymes of chlorophylls, which might be related to a high chlorophyll content (Hasanuzzaman et al., 2017; Alharby et al., 2020). Exogenous GSH supplied to drought or salt stressed-plant restored its reduced content of chlorophylls (Nahar et al., 2017; Zaki and Mohamed, 2018).

Osmoprotectant materials are accumulated in many plant species in response to various stress conditions. Our study showed significant positive changes in osmotic adjustment substances, especially soluble sugars and free proline under combined $\mathrm{DiW}+$ salt stress conditions (Table 2; Figure 2). Similarly, Abd ElMageed et al. (2018) and Alharby et al. (2020) observed a significant increase in the total soluble sugars and free proline contents of plants in DiW + salt treatments. In this study, the content of total soluble sugars increased significantly with DiW by $20 \%$ ( $80 \%$ of FC), while it decreased under DiW by $40 \%$ ( $60 \%$ of FC) throughout salt stressed-pepper growth, however, the free proline content increased significantly under both $\mathrm{DiW}$ regimes. However, a further increase in these leaf osmoprotective substances was observed using GSH application (Table 2; Figure 2). Proline is a compatible solute, which plays a crucial role in reducing stressinduced cell acidification and maintaining osmoregulation, and acts as an osmoprotectant (Hasegawa et al., 2000). It can prevent water loss or preserve leaf RWC (Abd El-Mageed et al., 2018). Respectively, Merwad et al. (2018) and Zaki et al. (2019), using the soybean and cowpea plants, reported the highest levels of proline and soluble sugars by exogenous GSH under drought and saline treatments.

The main components of the ascorbate (AsA)-glutathione (GSH) cycle; AsA and GSH are lowmolecular-weight (LMW) antioxidants that are synthesized within cell chloroplasts and play important roles as redox buffers to modulate processes involving in plant growth and development from mitosis and cell elongation to senescence and death (Kasote et al., 2015). These LMW antioxidants increased significantly with 
increasing DiW from 20 to $40 \%$ and further increased with the application of GSH to the salt-stressed chili pepper plants (Table 2; Figure 2). These compounds can activate gene expression associated with responses to biotic and abiotic stress conditions to increase the defense of sensitive plants (Kasote et al., 2015). AsA is generated during aerobic metabolism and is known to be the best molecule to detoxify $\mathrm{H}_{2} \mathrm{O}_{2}$, especially as a substrate of ascorbate peroxidase (APX), which is an essential enzyme for the AsA-GSH cycle, found in most compartments of the plant cell. It also helps regenerate the antioxidant pigments, carotenoids, and $\alpha$ tocopherol (Smirnoff and Wheeler, 2000). In this concern, the high activity of the antioxidant enzymes implicated in the AsA-GSH cycle is closely related to the increase in GSH content in response to DiW (Hasanuzzaman et al., 2017). The increase in endogenous AsA and GSH and their redox states supported the plant's tolerance to combined DiW + salinity stress (Alharby et al., 2020).

GSH is the most abundant LMW thiol in plants (Noctor et al., 2012). The sulphhydryl or thiol group $(-\mathrm{SH})$ of GSH can donate an electron to free radicals. Because of its abundance and potential for negative redox, GSH contributes strongly to the redox environment, allowing cells to maintain healthy reduced redox homeostasis (Alharby et al., 2020). GSH (reduced glutathione) is oxidized to the glutathyl anion radical (GS*). Two of the GS* can spontaneously bind to form glutathione disulphide (GSSG; oxydized glutathione), which can be recycled back to GSH by GR, which requires NADPH as reducing power. GSSG can form disulfides mixed with thiol-containing proteins. In this way, protein-bound GSH protects protein thiol groups from auto-oxidation to sulfonic acids (Hasanuzzaman et al., 2017). GSH acts as an antioxidant by suppressing ROS and is a participant in the AsA-GSH cycle, which eliminates harmful peroxides (Kasote et al., 2015). In a study conducted by Herbinger et al. (2002) using the Triticum aestivum plants, GSH concentrations are significantly increased in response to DiW up to $40 \%$ of the soil water capacity. Through the use of long-term DiW-suffered Vigna radiata plants, Sengupta et al. (2012) showed a decrease in activity and level of transcription of $\gamma$ glutamylcysteine synthetase in plant roots. This is incompatible with the hypothesis that tolerance to abiotic stress is associated with an increase in the level and activity of $\gamma$-glutamyl-cysteine synthetase with an increase in GSH and cysteine concentrations, as demonstrated under salt stress (Nazar et al., 2011).

The main role of alkaloid substances is generally linked to plant defense mechanisms against predators, in addition to the important ecological factors associated with them. However, the close relationship between alkaloids and the redox processes in the plants they contain strongly suggests that these compounds play a key role in protecting plants when they are under oxidative stress (Ramos-Valdivia et al., 2012). In the present study, a decrease in the water supply to the chili pepper plant root-zone significantly increased the total content of capsaicin in fruits. These results are close to those reported by Jaleel et al. (2007) who hypothesized that similar to phenolic compounds, an increase in the total content of indole alkaloid has been observed in shoots and roots of Catharanthus roseus under drought-induced stress. Additionally, higher production of alkaloids has been reported during in vitro and in vivo growth of Hypericum polyanthemum under drought stress through induction of ionic or osmotic stress (De-Matos et al., 2014). However, the addition of GSH as foliar application further increased the total content of alkaloid capsaicin in fruits (Table 2; Figure 2) to impose a protective effect (Kotebagilu et al., 2014) against tested stresses. In this study, GSH and alkaloid capsaicin functionally acted side-by-side in favor of the antioxidant defense system in the combined-stressed chili pepper plants. Capsaicin can function as an $\mathrm{H}_{2} \mathrm{O}_{2}$ scavenging system. As a result, the increase in GSH-induced capsaicin functionally acted side-by-side to minimize the effects of combined $\mathrm{DiW}+$ salt stress by scavenging ROS.

As shown in Table 2; Figure 2, endogenous GSH and AsA levels were significantly elevated from GSH applied exogenously to enrich the GSH-AsA cycle to raise its efficiency to detoxify species of oxygen radicals (ROS) to suppress oxidative stress in plants. This interpretation is in agreement with Nahar et al. (2017), reporting that a higher level of GSH in plants efficiently removes ROS and suppresses oxidative damage. They also added that an exogenous GSH can efficiently recycle AsA to eliminate ROS. GSH can attenuate the prohibited influences of ROS-stimulated oxidative stress. Besides, the use of GSH externally boosted other non-enzymatic components of the plant antioxidant system such as proline, phenolic compounds, and 
capsaicin (Table 2; Figure 2) that worked to prevent ROS-stimulated oxidative stress damage in stressed plants (Nahar et al., 2017) to help maintain homeostasis of cellular redox. The exogenous use of GSH maintains the homeostasis of GSH redox in plant leaves and helps to regulate synthesis and regeneration of GSH to meet its increasing need, thus boosting the chili pepper plant's tolerance to the adverse impacts of oxidative stress (Zhou et al., 2017).

An increase in $\mathrm{DiW}$ from $20 \%$ to $40 \%$ gradually reduced the leaf contents of macro-nutrients (e.g., nitrogen; $\mathrm{N}$, phosphorus; $\mathrm{P}$, potassium; $\mathrm{K}^{+}$, calcium; $\mathrm{Ca}^{2+}$, and magnesium; $\mathrm{Mg}^{2+}$ ) and micro-nutrients (e.g., iron; Fe, zinc; $\mathrm{Zn}$ and manganese; $\mathrm{Mn})$, as well as the ratio of $\mathrm{K}^{+} / \mathrm{Na}^{+}$, while the leaf contents of sodium $\left(\mathrm{Na}^{+}\right)$ and chloride $\left(\mathrm{Cl}^{-}\right)$were gradually increased in salt-stressed chili pepper plants compared to the control (Table 3; Figure 3). These results are confirmed by Zaki and Mohamed (2018) and Bekmirzaev et al. (2019) using faba bean and Tetragonia tetragonioides plants, respectively, under different stress conditions. However, GSH mitigated the adverse effects of DiW by increasing all investigated macro- and micro-nutrient contents and $\mathrm{K}^{+} / \mathrm{Na}^{+}$ratio, while $\mathrm{Na}^{+}$and $\mathrm{Cl}^{-}$contents were decreased significantly in chili pepper plants. These positive results were more pronounced with the $0.8 \mathrm{mM}$ GSH level. The positive role of GSH in improving plant ion contents under DiW may be due to its effect in modifying membrane permeability and improving osmotic tolerance and/or regulation of biochemical processes (Kattab, 2007). Zaki and Mohamed (2018) obtained similar results with exogenously-applied GSH, which prohibited the deleterious effect of salinity stress and increased both the mineral ion contents (e.g., $\mathrm{K}, \mathrm{Ca}, \mathrm{Mg}, \mathrm{P}$, and $\mathrm{N}$ ) and $\mathrm{K}^{+} / \mathrm{Na}^{+}$ratio, while $\mathrm{Na}^{+}$content was decreased compared to the control. Under stress conditions as in this study, an increased level of abscisic acid (ABA) has been reported to stimulate stomatal closure (Aliniaeifard et al., 2014). Under this condition (stomatal closure), the influx of water and nutrient ions dissolved in the water from roots to leaves will diminish due to the suppressed rate of transpiration, thus lowering the nutrient contents as shown in Table 3; Figure 3 . Table 2; Figure 2 displays that applying GSH markedly increased the level of AsA, which has been reported to reverse stomatal closure (Chen and Gallie, 2004). AsA localized in cellular walls plays a specific role in regulating stomatal dynamics through the action of dehydroascorbate (Fotopoulos et al., 2008). Thus, AsA scavenges ROS and prevents ABA-stimulated stomatal closure (Chen and Gallie, 2004), and ionic homeostasis will occur in the leaves. Besides, applying GSH considerably increased the osmoprotectant compounds like proline and soluble sugars, reinforcing the power of osmotic absorption and thus more water uptake along with the nutrients. Therefore, the increase in the contents of nutrients will occur as shown in this study (Table 3; Figure 3). In this study, there was a crucial role of different osmotic protective compounds (proline and soluble sugars) in plant tolerance to DiW because these compounds regulate a wide range of metabolic processes, including up-regulating ion transport and transpiration rate (Iqbal, 2018).

Changes in the features of leaf and stem anatomy that appeared in chili pepper plant as affecting by combined stress (DiW + salinity) may be attributed to the osmotic effect and deficiency of nutrients. The combined stress negative effects on anatomical features were due to defects that occurred in leaf midvein and blade and the decrease in stem diameter and its components, as well as due to plant cell plasmolysis and cell shrinkage due to lack of water due to stress. Barcelo et al. (1986) noted that one of the mechanisms of stress is the negative effects on cell elongation and wall plasticity. However, the foliar application of GSH, especially at $0.8 \mathrm{mM}$, alleviated the adverse effects of $\mathrm{DiW}+$ salt stress on all anatomical parameters of the leaf and stem of chili plants. This positive effect may be attributed to increased cell wall expansion along with higher turgor pressure by applying GSH (Munns and Termaat, 1986). In the present study, the foliar application of GSH maintained osmotic and ionic balance, thus improving the plant water status to resist $\mathrm{DiW}+$ salt stress and improving the anatomical features of the chili pepper plant. 
Table 5. Changes (\%) in plant growth, physiology, biochemistry, productivity, and water use efficiency relative to the control in chili pepper plants grown under saline soil $\left(\mathrm{EC}=6.74 \mathrm{dS} \mathrm{m}^{-1}\right)$ conditions. Three color scale heatmap, yellow as the midpoint of control and parameters with insignificant values compared to control, red for changes below control values, and green for changes over control values

\begin{tabular}{|c|c|c|c|c|c|c|}
\hline \multirow{3}{*}{ Parameters } & \multicolumn{6}{|c|}{ Treatments } \\
\hline & \multicolumn{3}{|c|}{ Irrigation regimes $(\mathrm{FC}, \%)$} & \multicolumn{3}{|c|}{ Glutathione treatments (mM) } \\
\hline & $\begin{array}{c}100 \\
\text { (control) }\end{array}$ & 80 & 60 & $\begin{array}{c}0 \\
\text { (control) }\end{array}$ & 0.4 & 0.8 \\
\hline Plant height & $\mathrm{a}$ & $-21.5 b$ & $-43.8 c$ & $\mathrm{C}$ & $+44.3 b$ & $+81.6 \mathrm{a}$ \\
\hline No. of branches plant ${ }^{-1}$ & $\mathrm{a}$ & $-18.6 b$ & $-40.4 \mathrm{c}$ & $\mathrm{C}$ & $+39.6 b$ & $+63.7 \mathrm{a}$ \\
\hline No. of branches plant ${ }^{-1}$ & $\mathrm{a}$ & $-18.2 b$ & $-39.3 c$ & $\mathrm{C}$ & $+21.3 b$ & $+37.8 \mathrm{a}$ \\
\hline Shoot fresh weight & $\mathrm{a}$ & $-18.0 \mathrm{~b}$ & $-35.5 c$ & $\mathrm{C}$ & $+27.5 b$ & $+60.3 \mathrm{a}$ \\
\hline Root fresh weight & $\mathrm{a}$ & $-26.6 b$ & $-48.3 c$ & $\mathrm{C}$ & $+41.0 \mathrm{~b}$ & $+94.0 \mathrm{a}$ \\
\hline Shoot dry weight & $\mathrm{a}$ & $-25.1 b$ & $-46.4 c$ & $\mathrm{C}$ & $+35.4 \mathrm{~b}$ & $+66.4 \mathrm{a}$ \\
\hline Root dry weight & $\mathrm{a}$ & $-22.9 b$ & $-44.3 c$ & $\mathrm{C}$ & $+41.6 b$ & $+96.6 \mathrm{a}$ \\
\hline No. of fruits plant ${ }^{-1}$ & $\mathrm{~b}$ & $+58.9 \mathrm{a}$ & $-52.4 \mathrm{c}$ & $\mathrm{C}$ & $+98.3 b$ & $+235 \mathrm{a}$ \\
\hline Fruit fresh weight & $\mathrm{b}$ & $+58.9 \mathrm{a}$ & $-51.7 c$ & $\mathrm{C}$ & $+102 b$ & $+241 \mathrm{a}$ \\
\hline Fruit dry weight & $\mathrm{b}$ & $+71.6 \mathrm{a}$ & $-46.6 c$ & $\mathrm{C}$ & $+109 \mathrm{~b}$ & $+258 \mathrm{a}$ \\
\hline Water use efficiency (WUE) & $\mathrm{b}$ & $+98.2 \mathrm{a}$ & $-19.7 c$ & $\mathrm{C}$ & $+98.8 b$ & $+237 \mathrm{a}$ \\
\hline SPAD chlorophyll index & $\mathrm{a}$ & $-11.5 b$ & $-20.6 c$ & $\mathrm{C}$ & $+4.4 b$ & $+11.1 \mathrm{a}$ \\
\hline Soluble sugars content & $\mathrm{a}$ & $+7.4 \mathrm{a}$ & $+11.5 \mathrm{a}$ & $\mathrm{C}$ & $+14.7 \mathrm{~b}$ & $+30.2 \mathrm{a}$ \\
\hline Proline content & $\mathrm{c}$ & $+18.9 \mathrm{~b}$ & $+28.9 \mathrm{a}$ & $\mathrm{A}$ & $+5.8 \mathrm{a}$ & $+7.8 \mathrm{a}$ \\
\hline Phenolics content & $\mathrm{c}$ & $+46.5 b$ & $+55.8 \mathrm{a}$ & $\mathrm{C}$ & $+7.7 \mathrm{~b}$ & $+25.0 \mathrm{a}$ \\
\hline Capsaicin content & $\mathrm{c}$ & $+9.0 \mathrm{~b}$ & $+19.1 \mathrm{a}$ & $\mathrm{C}$ & $+14.0 \mathrm{~b}$ & $+25.6 \mathrm{a}$ \\
\hline Ascorbate content & $\mathrm{c}$ & $+48.1 \mathrm{~b}$ & $+106 \mathrm{a}$ & $\mathrm{C}$ & $+8.0 \mathrm{~b}$ & $+18.1 \mathrm{a}$ \\
\hline Glutathione content & $\mathrm{c}$ & $+64.6 b$ & $+149 a$ & $\mathrm{C}$ & $+26.4 b$ & $+55.0 \mathrm{a}$ \\
\hline $\mathrm{N}$ content & $\mathrm{a}$ & $-10.7 b$ & $-15.7 b$ & $\mathrm{C}$ & $+21.6 b$ & $+37.2 \mathrm{a}$ \\
\hline P content & $\mathrm{a}$ & $-16.1 b$ & $-32.3 c$ & $\mathrm{C}$ & $+85.7 \mathrm{~b}$ & $+171 \mathrm{a}$ \\
\hline $\mathrm{Ca}^{2+}$ content & $\mathrm{a}$ & $-6.4 b$ & $-24.8 c$ & $\mathrm{C}$ & $+13.6 b$ & $+27.0 \mathrm{a}$ \\
\hline $\mathrm{Mg}^{2+}$ content & $\mathrm{a}$ & $-7.3 b$ & $-13.5 c$ & $\mathrm{~B}$ & $+0.6 \mathrm{~b}$ & $+3.4 a$ \\
\hline $\mathrm{Fe}^{2+}$ content & $\mathrm{a}$ & $-8.0 b$ & $-17.7 \mathrm{c}$ & $\mathrm{C}$ & $+12.0 \mathrm{~b}$ & $+26.6 \mathrm{a}$ \\
\hline $\mathrm{Zn}^{2+}$ content & $\mathrm{a}$ & $-17.1 b$ & $-26.8 c$ & $\mathrm{C}$ & $+280 \mathrm{~b}$ & $+470 \mathrm{a}$ \\
\hline $\mathrm{Mn}^{2+}$ content & $\mathrm{a}$ & $-7.7 b$ & $-20.5 c$ & $\mathrm{C}$ & $+171 \mathrm{~b}$ & $+286 a$ \\
\hline $\mathrm{K}^{+}$content & $\mathrm{a}$ & $-13.2 b$ & $-32.9 c$ & $\mathrm{C}$ & $+18.9 \mathrm{~b}$ & $+38.2 \mathrm{a}$ \\
\hline $\mathrm{Na}^{+}$content & $\mathrm{b}$ & $+9.1 \mathrm{a}$ & $+13.6 \mathrm{a}$ & A & $-7.7 b$ & $-19.2 c$ \\
\hline $\mathrm{Cl}^{-}$content & c & $+9.5 b$ & $+25.5 \mathrm{a}$ & A & $-9.9 b$ & $-23.3 c$ \\
\hline $\mathrm{K}^{+} / \mathrm{Na}^{+}$ratio & $\mathrm{a}$ & $-18.5 b$ & $-40.2 c$ & $\mathrm{C}$ & $+29.8 b$ & $+71.1 \mathrm{a}$ \\
\hline
\end{tabular}

From the results of this study and Table 5, chili pepper plants can be irrigated with $80 \%$ of soil field capacity, saving about $20 \%$ of water with elevating the water use efficiency and increasing the obtained fruit yield components, although the growth of plants decreased. These positive results were connected with exogenous application of $0.8 \mathrm{mM} \mathrm{GSH}$, which support growth and productivity of chili pepper plants under $20 \%$ shortage of irrigation water through the significant improvement in physiology and biochemistry, including antioxidants and nutrient homeostasis.

\section{Conclusions}

Our results showed that $0.8 \mathrm{mM}$ glutathione (GSH) applied to leaves improved the growth, physiobiochemical, and anatomical performances of chili pepper plants grown under drought and salinity. Damage 
from these stresses was mitigated with the use of GSH by maintaining the osmotic homeostasis by regulating the proline, soluble sugars, and $\mathrm{K}^{+}$contents to maximize the plant's water status and healthy metabolic processes. Plant defense system components such as proline, phenolic compounds, and the alkaloid capsaicin have also been improved, as well as nutrient homeostasis and anatomical features. Our study suggested the use of $0.8 \mathrm{mM}$ GSH for satisfying growth and yield with higher WUE of chili pepper plants grown under saltaffected conditions with deficit irrigation.

\section{Authors' Contributions}

Conceptualization: OAAIA, GFM, AAAL; Methodology: OAAIA, GFM, HAA; Validation: MMR; Formal analysis: OAAIA, GFM, HAA; Investigation: OAAIA, GFM, HAA; Data curation: OAAIA, GFM, HAA, AAAL; Funding acquisition: AAAL; Project administration: AAAL. Writing: OAAIA, GFM, HAA; Review and editing; MMR, AAAL. All authors read and approved the final manuscript.

\section{Acknowledgements}

The authors would like to extend their sincere appreciation to their Institutions and acknowledge the Taif University Researchers Supporting Project number (TURSP-2020/72), Taif University, Taif, Saudi Arabia.

\section{Conflict of Interests}

The authors declare that there are no conflicts of interest related to this article.

\section{References}

Abd El-Mageed TA, Rady MM, Taha RS, Abdelaziz SA, Simpson CR, Semida WM (2020). Effects of integrated use of residual sulfur-enhanced biochar with effective microorganisms on soil properties, plant growth and short-term productivity of Capsicum annuum under salt stress. Scientia Horticulturae 261:108930. https://doi.org/10.1016/j.scienta.2019.108930

Abd El-Mageed TA, Semida WM, Taha RS, Rady MM (2018). Effect of summer-fall deficit irrigation on morphophysiological, anatomical responses, fruit yield and water use efficiency of cucumber under salt affected soil. Scientia Horticulturae 237:148-155. https://doi.org/10.1016/j.scienta.2018.04.014

Abdel Latef AA, Kordrostami M, Zakir A, Zaki H, Saleh OM (2019a). Eustress with $\mathrm{H}_{2} \mathrm{O}_{2}$ facilitates plant growth by improving tolerance to salt stress in two wheat cultivars. Plants 8:303. https://doi.org/10.3390/plants8090303

Abdel Latef AA, Abu Alhmad MFA, Ahmad S (2017a). Foliar application of fresh moringa leaf extract overcomes salt stress in fenugreek (Trigonella foenum-graecum) plants. Egyptian Journal of Botany 57:157-179. https:// doi.org/10.21608/ejbo.2017.317.1011

Abdel Latef AA, Alhmad MFA, Abdelfattah KE (2017b). The possible roles of priming with $\mathrm{ZnO}$ nanoparticles in mitigation of salinity stress in lupine (Lupinus termis) plants. Journal of Plant Growth Regulation 36:60-70. https://doi.org/10.1007/s00344-016-9618-X

Abdel Latef AA, Alhmad MFA, Kordrostami M, Abo-Baker A-B A-E, Zakir A (2020). Inoculation with Azospirillum lipoferum or Azotobacter chroococcum reinforces maize growth by improving physiological activities under saline conditions. Journal of Plant Growth Regulation 39:1293-1306. https://doi.org/10.1007/s00344-020$10065-9$ 
Abdel Latef AA, Chaoxing H (2014). Does inoculation with Glomus mosseae improve salt tolerance in pepper plants? Journal of Plant Growth Regulation 33:64-653. https://doi.org/10.1007/s00344-014-9414-4

Abdel Latef AA, Mostofa MG, Rahman MM, Abdel-Farid IB, Tran L-SP (2019b). Extracts from yeast and carrot roots enhance maize performance under seawater-induced salt stress by altering physio-biochemical characteristics of stressed plants. Journal of Plant Growth Regulation 38:966-979. https://doi.org/10.1007/s00344-018-9906-8

Abdel Latef AA, Srivastava AK, Abdel-sadek MS, Kordrostam M, Tran L-SP (2018). Titanium dioxide nanoparticles improve growth and enhance tolerance of broad bean plants under saline conditions. Land Degradation $\&$ Development 29:1065-1073. https://doi.org/10.1002/ldr.2780

Abdel Latef AA, Srivastava AK, Saber H, Alwaleed EA, Tran L-SP (2017c). Sargassum muticum and Jania rubens regulate amino acid metabolism to improve growth and alleviate salinity in chickpea. Scientific Reports 7:10537. https://doi.org/10.1038/s41598-017-07692-W

Abid M, Zhang YJ, Li Z, Bai DF, Zhong YP, Fang JB (2020). Effect of salt stress on growth, physiological and biochemical characters of four kiwifruit genotypes. Scientia Horticulturae 271:109473. https://doi.org/10.1016/j.scienta.2020.109473

Alharby HF, Alzahrani YM, Rady MM (2020). Seeds pretreatment with zeatins or maize grain-derived organic biostimulant improved hormonal contents, polyamine gene expression, and salinity and drought tolerance of wheat. International Journal of Agriculture and Biology 24(4):714-724. https://doi.org/10.17957/IJAB/15.1491

Aliniaeifard S, Malcolm Matamoros P, van Meeteren U (2014). Stomatal malfunctioning under low vapor pressure deficit (VPD) conditions: Induced by alterations in stomatal morphology and leaf anatomy or in the ABA signaling? Physiologia Plantarum 152:688-699. https://doi.org/10.1111/ppl.12216

Augusto TG, Carloswild A (1973). A new method for the determination of capsaicin in capsicum fruits. Journal of Food Science 38(2):342-344. https://doi.org/10.1111/j.1365-2621.1973.tb01422.x

Barcelo J, Poschenrieder CH, Andren I, Gunse B (1986). Cadmium induced decrease of water stress resistance in bush bean plants (Phaseolus vulgaris cv. Contender). Plant Physiology 125:17-25. https://doi.org/10.1016/S01761617(86)80239-5

Bates LS, Waldeen RP, Teare ID (1973). Rapid determination of free proline for water stress studies. Plant and Soil 39:205-207. https://doi.org/10.1007/BF00018060

Bekmirzaev G, Beltrao J, Ouddane B (2019). Effect of irrigation water regimes on yield of Tetragonia tetragonioides. Agriculture 9:22. https://doi.org/10.3390/agriculture9010022

Ben-Gal A, Ityel E, Dudley L, Cohen S, Yermiyahu U, Presnov E, Zigmond L, Shani U (2008). Effect of irrigation water salinity on transpiration and on leaching requirements: A case study for bell peppers. Agricultural Water Management 95:587-597. https://doi.org/10.1016/j.agwat.2007.12.008

Caliskan O, Radusiene J, Temizel KE, Staunis Z, Cirak C, Kurt D, Odabas MS (2017). The effects of salt and drought stress on phenolic accumulation in greenhouse-grown Hypericum pruinatum. Italian Journal of Agronomy 12(918):271-275. https://doi.org/10.4081/ija.2017.918

Cao F, Fu M, Wang R, Diaz-Vivancos P, Hossain MA (2017). Exogenous glutathione-mediated abiotic stress tolerance in plants. In: Hossain MA, Mostofa MG, Diaz-Vivancos P, Burritt DJ, Fujita M, Tran LSP (Eds). Glutathione in plant growth, development, and stress tolerance, 1st edn. Springer, Basel, pp 171-194. https://doi.org/10.1007/978-3-319-66682-2_8

Chen JH, Jiang HW, Hsieh EJ, Chen HY, Chien CT, Hsieh HL, Lin TP (2012). Drought and salt stress tolerance of an Arabidopsis glutathione S-transferase U17 knockout mutant are attributed to the combined effect of glutathione and abscisic acid. Plant Physiology 158:340-351. https://doi.org/10.1104/pp.111.181875

Chen Z, Gallie, DR (2004). The ascorbic acid redox state controls guard cell signaling and stomatal movement. The Plant Cell 16:1143-1162. https://doi.org/10.1105/tpc.021584

Correia MJ, Coelho D, David MM (2001). Response to seasonal drought in three cultivars of Ceratonia siliqua; leaf growth and water relation. Tree Physiology 21:645-653. https://doi.org/10.1093/treephys/21.10.645

Dahnke WC, Whitney DA (1988). Measurement of soil salinity. In: Dahnke WC (Ed.), Recommended Chemical Soil Test Procedures for the North Central Region, 499. North Central Regional Publication 221. North Dakota Agricultural Experiment Station Bulletin, pp 32-34.

Dai YJ, Shen ZG, Liu Y, Wang LL, Hannaway D, Lu HF (2009). Effects of shade treatments on the photosynthetic capacity, chlorophyll fluorescence, and chlorophyll content of Tetrastigma hemsleyanum Diels et Gilg. Environmental and Experimental Botany 65:177-182. https://doi.org/10.1016/j.envexpbot.2008.12.008 
De-Matos NJ, Bertodo LOO, Da-Rosa LMG, Von-Poser GL, Rech SB (2014). Stress induction of valuable secondary metabolites in Hypericum polyanthemum acclimatized plants. South African Journal Botany 94:182-189. https://doi.org/10.1016/j.sajb.2014.06.014

Desoky EM, Elrys AS, Rady MM (2019). Integrative moringa and licorice extracts application improves Capsicum annuum fruit yield and declines its contaminant contents on a heavy metals-contaminated saline soil. Ecotoxicology and Environmental Safety 169:50-60. https://doi.org/10.1016/j.ecoenv.2018.10.117

El-Beltagi HS, Mohamed HI, Sofy MR (2020). Role of ascorbic acid, glutathione and proline applied as singly or in sequence combination in improving chickpea plant through physiological change and antioxidant defense under different levels of irrigation intervals. Molecules 25:1702. https://doi.org/10.3390/molecules25071702

Farooq M, Gogoi N, Hussain M, Barthakur S, Paul S, Bharadwaj N, ... Siddique KHM (2017). Effects, tolerance mechanisms and management of salt stress in grain legumes. Plant Physiology and Biochemistry 118:199-217. https://doi.org/10.1016/j.plaphy.2017.06.020

Fotopoulos V, De Tullio MC, Barnes J, Kanellis AK (2008). Altered stomatal dynamics in ascorbate oxidase overexpressing tobacco plants suggest a role for dehydroascorbate signalling. Journal of Experimental Botany 59:729737. https://doi.org/10.1093/jxb/erm359

Foyer CH, Noctor G (2005a). Redox homeostasis and antioxidant signaling: a metabolic interface between stress perception and physiological responses. Plant Cell 17:1866-1875. https://doi.org/10.1016/j.plaphy.2017.06.020

Foyer CH, Noctor G (2005b). Oxidant and antioxidant signaling in plants: a re-evaluation of the concept of oxidative stress in a physiological context. Plant, Cell \& Environment 28:1056-1071. https://doi.org/10.1111/j.13653040.2005.01327.x

Gill SS, Tuteja N (2010). Review reactive oxygen species and antioxidant machinery in abiotic stress tolerance in crop plants. Plant Physiology and Biochemistry 48(12):909-930. https://doi.org/10.1016/j.plaphy.2010.08.016

Guang-Cheng S, Zhan-Yu Z, Na L, Shuang-En Y, Weng-Gang X (2008). Comparative effects of deficit irrigation (DI) and partial rootzone drying (PRD) on soil water distribution, water use, growth and yield in greenhouse grown hot pepper. Scientia Horticulturae 119:11-16. https://doi.org/10.1016/j.scienta.2008.07.001

Hasanuzzaman M, Nahar K, Anee TI, Fujita M (2017). Glutathione in plants: biosynthesis and physiological role in environmental stress tolerance. Physiology and Molecular Biology of Plants 23(2):249-268. https://doi.org/10.1007/s12298-017-0422-2

Hasegawa PM, Bressan RA, Zhu JK, Bohnert HJ (2000). Plant cellular and molecular responses to high salinity. Annual Review of Plant Physiology and Plant Molecular Biology 51:463-499. https://doi.org/10.1146/annurev.arplant.51.1.463

Herbinger K, Tausz M, Wonisch A, Soja G, Sorger A, Grill D (2002). Complex interactive effects of drought and ozone stress on the antioxidant defence systems of two wheat cultivars. Plant Physiology and Biochemistry 40:691-696. https://doi.org/10.1016/S0981-9428(02)01410-9

Higinbotham N, Bud E, Foster RJ (1967). Mineral ion contents and cell trans-membranes electro-potentials of peas and oat seedling tissues. Plant Physiology 24:37-46. https://doi.org/10.1104/pp.42.1.37

Hong-Bo S, Li-Ye C, Cheruth AJ, Chang-Xing Z (2008). Water deficit stress induced anatomical changes in higher plants. Current Research in Biology (Comptes Rendus Biologies) 331:215-225. https://doi.org/10.1016/j.crvi.2008.01.002

Horie T, Brodsky DE, Costa A, Kaneko T, Schiavo FL, Katsuhara M, Schroeder JI (2011). K+ transport by the OsHKT2; 4 transporter from rice with a typical $\mathrm{Na}+$ transport properties and competition in permeation of $\mathrm{K}+$ over $\mathrm{Mg} 2+$ and Ca2+ ions. Plant Physiology 156:1493-1507. https://doi.org/10.1104/pp.110.168047

Hussain M, Farooq S, Hasan W, Ul-Allah S, Tanveer M, Farooq M, Nawaz A (2018). Drought stress in sunflower: Physiological effects and its management through breeding and agronomic alternatives. Agricultural Water Management 201:152-167. https://doi.org/10.1016/j.agwat.2018.01.028

Iqbal MJ (2018). Role of osmolytes and antioxidant enzymes for drought tolerance in wheat. Global Wheat Production, Shah Fahad, Abdul Basir and Muhammad Adnan, IntechOpen. http://dx.doi.org/10.5772/intechopen.75926

Irigoyen JJ, Emerich DW, Sanchez-Diaz M (1992). Water stress induced changes in the concentrations of proline and total soluble sugars in nodulated alfalfa (Medicago sativa) plants. Plant Physiology 8:455-460. https://doi.org/10.1111/j.1399-3054.1992.tb08764.x 
Ismail SM (2010). Influence of deficit irrigation on water use efficiency and bird pepper production (Capsicum annuum L.). JKAU: Meteorology, Environment and Arid Land Agriculture Sciences 21:29-43. https://doi.org/10.4197/met.21-2.3

Jackson ML (1967). Soil chemical analysis. Prentice Hall of India Pvt. Ltd, New Delhi, India pp 144-197 and 326-338. https://doi.org/10.1002/jpln.19590850311

Jaleel CA, Manivannan P, Sankar B, Kishorekumar A, Gopi R, Somasundaram R, Panneerselvam R (2007). Water deficit stress mitigation by calcium chloride in Catharanthus roseus: effects on oxidative stress, proline metabolism and indole alkaloid accumulation. Colloids and Surfaces B: Biointerfaces 60:110-116. https://doi.org/10.1016/j.colsurfb.2007.06.006

Kasote DM, Katyare SS, Hegde MV, Bae H (2015). Significance of antioxidant potential of plants and its relevance to therapeutic applications. International Journal of Biological Sciences 11(8):982-991. https://doi.org/10.7150/ijbs.12096

Kattab H (2007). Role of glutathione and polyadenylic acid on the oxidative defense systems of two different cultivars of canola seedlings grown under saline condition. Australian Journal of Basic and Applied Sciences 1:323-332.

Kopta T, Sekara A, Pokluda R, Ferby V, Caruso G (2020). Screening of chilli pepper genotypes as a source of capsaicinoids and antioxidants under conditions of simulated drought stress. Plants 9(364):1-17. https://doi.org/10.3390/plants9030364

Kotebagilu NP, Palvai VR, Urooj A (2014). Protective effect of selected medicinal plants against hydrogen peroxide induced oxidative damage on biological substrates. International Journal of Medicinal Chemistry 12:1-7. https://doi.org/10.1155/2014/861084

Kpinkoun JK, Amoussa AM, Mensah ACG, Komlan FA, Kinsou E, Lagnika L, Gandonou CB (2019). Effect of salt stress on flowering, fructification and fruit nutrients concentration in a local cultivar of chili pepper (Capsicum frutescens L.). International Journal of Plant Physiology and Biochemistry 11(1):1-7. https://doi.org/10.5897/IJPPB2019.0284

Lee SKD (2006). Hot pepper response to interactive effects of salinity and boron. Plant, Soil and Environment 52:227233. https://doi.org/10.17221/3433-PSE

Mardani S, Tabatabaei SH, Pessarakli M, Zareabyaneh H (2017). Physiological responses of pepper plant (Capsicum annuum L.) to drought stress. Journal of Plant Nutrition 40(10):1453-1464. https://doi.org/10.1080/01904167.2016.1269342

Merwad AMA, Desoky EM, Rady MM (2018). Response of water deficit-stressed Vigna unguiculata performances to silicon, proline or methionine foliar application. Scientia Horticulturae 228:132-144. https://doi.org/10.1016/j.scienta.2017.10.008

Munns R (2002). Comparative physiology of salt and water stress. Plant, Cell and Environment 25:239-250. https://doi.org/10.1046/j.0016-8025.2001.00808.x

Munns R, Termaat A (1986). Whole-plant responses to salinity. Australian Journal of Plant Physiology 13:143-160. https://doi.org/10.1071/PP9860143

Nahar K, Hasanuzzaman M, Alam MM, Fujita M (2017). Glutathione-induced drought stress tolerance in mung bean: coordinated roles of the antioxidant defence and methylglyoxal detoxification systems. AoB Plants 1(7):plv069. https://doi.org/10.1093/aobpla/plv069

Nassar MA, El-Sahhar KF (1998). Botanical Preparations and Microscopy (Microtechnique). Academic Bookshop. Dokki. Giza, Egypt, pp 219 (In Arabic)

Nazar R, Iqbal N, Syeed S, Khan NA (2011). Salicylic acid alleviates decreases in photosynthesis under salt stress by enhancing nitrogen and sulfur assimilation and antioxidant metabolism differentially in two mungbean cultivars. Journal of Plant Physiology 168:807-815. https://doi.org/10.1016/j.jplph.2010.11.001

Noctor G, Mhamdi A, Chaouch S, Han Y, Neukermans J, Marquez-Garcia B, ... Foyer C (2012). Glutathione in plants: An integrated overview. Plant, Cell and Environment 35(2):454-484. https://doi.org/10.1111/j.13653040.2011.02400.x

Osman MS, Badawy AA, Osman AI, Abdel Latef AAH (2020) Ameliorative impact of an extract of the halophyte Arthrocnemum macrostachyum on growth and biochemical parameters of soybean under salinity stress. Journal of Plant Growth Regulation https://doi.org/10.1007/s00344-020-10185-2 
Patil VC, Al-Gaadi KA, Wahb-Allah MA, Saleh AM, Marey SA, Samdani MS, Abbas ME (2014). Use of saline water for greenhouse bell pepper (Capsicum annuum) production. American Journal of Agricultural and Biological Sciences 9(2):208-217. https://doi.org/10.3844/ajabssp.2014.208.217

Phimchan P, Techawongstien S (2012). Impact of drought stress on the accumulation of capsaicinoids in capsicum cultivars with different initial capsaicinoid levels. HortScience 47(9):1204-1209. https://doi.org/10.21273/HORTSCI.47.9.1204

Pinheiro C, Chaves MM (2011). Photosynthesis and drought: can we make metabolic connections from available data? Journal of Experimental Botany 62:869-882. https://doi.org/10.1093/jxb/erq340

Rady MM (2012). A novel organo-mineral fertilizer can mitigate salinity stress effects for tomato production on reclaimed saline soil. South African Journal of Botany 81:8-14. https://doi.org/10.1016/j.sajb.2012.03.013

Rady MM, Belal HEE, Gadallah FM, Semida WM (2020). Selenium application in two methods promotes drought tolerance in Solanum lycopersicum plant by inducing the antioxidant defense system. Scientia Horticulturae 266:109290. https://doi.org/10.1016/j.scienta.2020.109290

Rady MM, Elrys AS, Abo El-Maati MF, Desoky EM (2019). Interplaying roles of silicon and proline effectively improve salt and cadmium stress tolerance in Phaseolus vulgaris plant. Plant Physiology and Biochemistry 139:558-568. https://doi.org/10.1016/j.plaphy.2019.04.025

Rady MM, Hemida KA (2016). Sequenced application of ascorbate-proline-glutathione improves salt tolerance in maize seedlings. Ecotoxicology and Environmental 133:252-259. https://doi.org/10.1016/j.ecoenv.2016.07.028

Ramos-Valdivia AC, Huerta-Heredia AA, Trejo Tapia G, Cerda-García-Rojas CM (2012). Secondary metabolites as nonenzymatic plant protectors from oxidative stress. In: Oxidative Stress in Plants: Causes, Consequences and Tolerance, Anjum NA, Umar S, Ahmad A (Eds.) (NewDelhi: IK International Publishers), pp 413-441.

Sabetta W, Paradiso A, Paciolla C, de Pinto MC (2017). Chemistry biosynthesis, and antioxidative function of glutathione in plants. In: Hossain MA, Mostofa MG, Diaz-Vivancos P, Burritt DJ, Fujita M, Tran LSP (Eds). Glutathione in plant growth, development, and stress tolerance, 1st edn. Springer, Basel, pp 1-27. https://doi.org/10.1007/978-3-319-66682-2_1

Sengupta S, Bisson WH, Mathew LK, Kolluri SK, Tanguay RL (2012). Alternate glucocorticoid receptor ligand binding structures influence outcomes in an in vivo tissue regeneration model. Comparative biochemistry and physiology. Comparative Biochemistry and Physiology - Part C: Toxicology \& Pharmacology 156(2):121-129. https://doi.org/10.1016/j.cbpc.2012.05.003

Shahid SA, Zaman M, Heng L (2018). Soil salinity: historical perspectives and a world overview of the problem. In: Guideline for Salinity Assessment, Mitigation and Adaptation Using Nuclear and Related Techniques (Springer, Cham), pp 43-53. https://doi.org/10.1007/978-3-319-96190-3_2

Singh R, Singh S, Parihar P, Mishra RK, Tripathi DK, Singh VP, Chauhan DK, Prasad SM (2016). Reactive Oxygen Species (ROS): Beneficial companions of plant's developmental processes. Frontiers in Plant Science 7:1299. https://doi.org/10.3389/fpls.2016.01299

Singleton VL, Rossi JA (1965). Colorimetry of total phenolics with phosphomolybdic-phosphotungstic acid reagents. American Journal of Enology and Viticulture 16:144-158.

Smirnoff N, Wheeler GL (2000). Ascorbic acid in plants: Biosynthesis and function. Critical Reviews in Plant Sciences 9:267-290. https://doi.org/10.1080/10409230008984166

Sohag AAM, Tahjib-Ul-Arif M, Polash MAS, Chowdhury MB, Afrin S, Burritt DJ, ... Hossain MA (2020). Exogenous glutathione-mediated drought stress tolerance in rice (Oryza sativa L.) is associated with lower oxidative damage and favorable ionic homeostasis. Iranian Journal of Science and Technology, Transaction A: Science 44:955971. https://doi.org/10.1007/s40995-020-00917-0

Srivalli S, Khanna-Chopra R (2008). Role of glutathione in abiotic stress tolerance. In: Khan NA, Singh S, Umar S (Eds). Sulfur assimilation and abiotic stress in plants. Springer, Berlin, pp 207-225. https://doi.org/10.1016/B978-012-799963-0.00005-8

Taha RS, Alharby HF, Bamagoos AA, Medani RA, Rady MM (2020). Elevating tolerance of drought stress in Ocimum basilicum using pollen grains extract; a natural biostimulant by regulation of plant performance and antioxidant defense system. South African Journal of Botany 128:42-53. https://doi.org/10.1016/j.sajb.2019.09.014

Taha SS, Mohamed AM, Rady MM (2018). Effect of exogenous $\alpha$-Tocopherol on sweet pepper plants irrigated by diluted sea water. Journal of Agricultural Studies 6(1):25-46. https://doi.org/10.5296/jas.v6i1.12365 
Tester M, Bacic A (2005). Abiotic stress tolerance in grasses. From model plants to crop plants. Plant Physiology 137:791793. https://doi.org/10.1104/pp.104.900138

Vernoux T, Wilson RC, Seeley KA, Reichheld JP, Muroy S, Brown S, ... Inze D (2000). The root meristem less cadmium sensitive 2 gene defines a glutathione-dependent pathway involved in initiation and maintenance of cell division during postembryonic root development. The Plant Cell 12:97-110. https://doi.org/10.1105/tpc.12.1.97

Wilde SA, Corey RB, Lyer JG, Voigt GK (1985). Soil and plant analysis for tree culture. Oxford and IBM Publishers, New Delhi, India, 3rd ed, pp 93-106.

Yang H, Shukla MK, Mao X, Kang S, Du T (2019). Interactive regimes of reduced irrigation and salt stress depressed tomato water use efficiency at leaf and plant scales by affecting leaf physiology and stem sap flow. Frontiers in Plant Science 10:160. https://doi.org/10.3389/fpls.2019.00160

Zaki SS, Belal EEE, Rady MM (2019). Cyanobacteria and glutathione applications improve productivity, nutrient contents, and antioxidant systems of salt-stressed soybean plant. International Letters of Natural Sciences 76:7285. https://doi.org/10.18052/www.scipress.com/ILNS.76.72

Zaki SS, Mohamed GF (2018). Alleviating effects of ascorbic acid and glutathione for faba bean plants irrigated with saline water. Journal of Plant Sciences 2(2):80-94. https://doi.org/10.25177/JPS.2.2.3

Zhou Y, Wen ZL, Zhang JW, Chen XJ, Cui JX, Xu W, Liu HY (2017). Exogenous glutathione alleviates salt induced oxidative stress in tomato seedlings by regulating glutathione metabolism, redox status, and the antioxidant system. Scientia Horticulturae 220:90-101. http://dx.doi.org/10.1016/j.scienta.2017.02.021
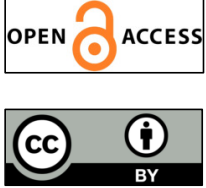

The journal offers free, immediate, and unrestricted access to peer-reviewed research and scholarly work. Users are allowed to read, download, copy, distribute, print, search, or link to the full texts of the articles, or use them for any other lawful purpose, without asking prior permission from the publisher or the author.

License - Articles published in Notulae Botanicae Horti Agrobotanici Cluj-Napoca are Open-Access, distributed under the terms and conditions of the Creative Commons Attribution (CC BY 4.0) License. (c) Articles by the authors; UASVM, Cluj-Napoca, Romania. The journal allows the author(s) to hold the copyright/to retain publishing rights without restriction. 